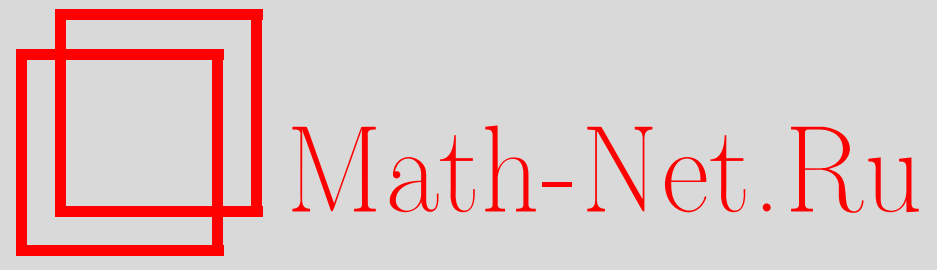

А. В. Калинкин, Вероятность остановки на границе случайного блуждания в четверти плоскости и ветвящийся процесс с взаимодействием частиц, Теория вероятн. и ее примен., 2002, том 47, выпуск $3,452-474$

DOI: https://doi.org/10.4213/tvp3676

Использование Общероссийского математического портала MathNet.Ru подразумевает, что вы прочитали и согласны с пользовательским соглашением

http: //www . mathnet.ru/rus/agreement

Параметры загрузки :

IP: 35.173 .219 .12

26 апреля 2023 г., $17: 26: 34$

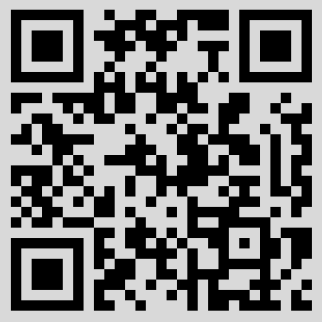




\section{ВЕРОЯТНОСТЬ ОСТАНОВКИ НА ГРАНИЦЕ СЛУЧАЙНОГО БЛУЖДАНИЯ В ЧЕТВЕРТИ ПЛОСКОСТИ И ВЕТВЯЩИЙСЯ ПРОЦЕСС С ВЗАИМОДЕЙСТВИЕМ ЧАСТИЦ}

Получены простые интегральные представления для вероятности остановки в точке границы случайного блуждания на целочисленной решетке четверти плоскости при различных предположениях о распределении скачка блуждания. Применяется предложенный в работах [18], [19] метод экспоненциальной производящей функции для решения стационарной первой (обратной) системы дифференциальных уравнений Колмогорова.

Ключевые слова и фразы: вероятность остановки случайного блуждания, ветвящийся процесс, экспоненциальная производящая функция, уравнение в частных производных гиперболического типа, задача Дарбу-Пикара, точные решения, многочлены Чебышева.

1. Случайное блуждание $S_{n}$ в четверти плоскости. На множестве состояний $\mathbf{N}^{2}=\left\{\left(\alpha_{1}, \alpha_{2}\right), \alpha_{1}, \alpha_{2}=0,1, \ldots\right\}$ рассматривается однородное случайное блуждание $S_{n}, n=0,1, \ldots$ Переходные вероятности за $n$ шагов обозначим $P_{\left(\beta_{1}, \beta_{2}\right)}^{\left(\alpha_{1}, \alpha_{2}\right)}(n)$. Пусть переходные вероятности за один шаг равны:

$$
\mathbf{P}\left\{S_{n+1}=\left(\beta_{1}, \beta_{2}\right) \mid S_{n}=\left(\alpha_{1}, \alpha_{2}\right)\right\}=p_{\beta_{1}-\alpha_{1}+1, \beta_{2}-\alpha_{2}+1}
$$

если $\alpha_{1}>0, \alpha_{2}>0, \beta_{1}-\alpha_{1}+1 \geqslant 0, \beta_{2}-\alpha_{2}+1 \geqslant 0$;

$$
\mathbf{P}\left\{S_{n+1}=\left(\alpha_{1}, \alpha_{2}\right) \mid S_{n}=\left(\alpha_{1}, \alpha_{2}\right)\right\}=1
$$

если $\alpha_{1}=0$ или $\alpha_{2}=0$. Здесь задано распределение вероятностей

$$
\left\{p_{\gamma_{1} \gamma_{2}} \geqslant 0,\left(\gamma_{1}, \gamma_{2}\right) \in \mathbf{N}^{2} ; \quad \sum_{\gamma_{1}, \gamma_{2}=0}^{\infty} p_{\gamma_{1} \gamma_{2}}=1, p_{11}=0\right\} \text {. }
$$

*Московский государственный технический университет им. Н.Э. Баумана, кафедра высшей математики, 2-я Бауманская ул., 5, 107005 Москва, Россия; http://www.bmstu.ru/facult/fn/kalin/kalinkin.htm 
На рис. 1 , случай а), изображены возможные скачки случайного блуждания $S_{n}$ из состояния $\left(\alpha_{1}, \alpha_{2}\right)$. Введем производящую функцию

$$
h\left(s_{1}, s_{2}\right)=\sum_{\gamma_{1}, \gamma_{2}=0}^{\infty} p_{\gamma_{1} \gamma_{2}} s_{1}^{\gamma_{1}} s_{2}^{\gamma_{2}} .
$$

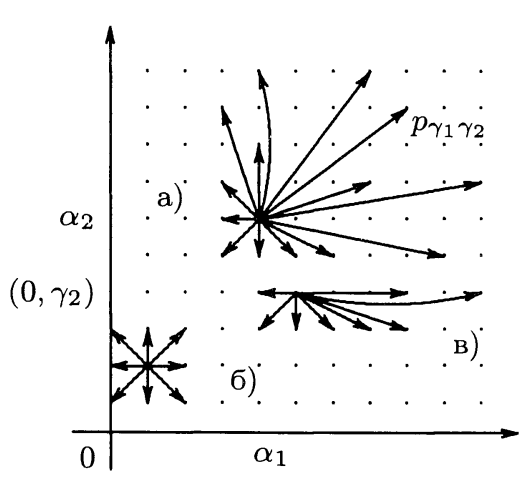

Рис. 1

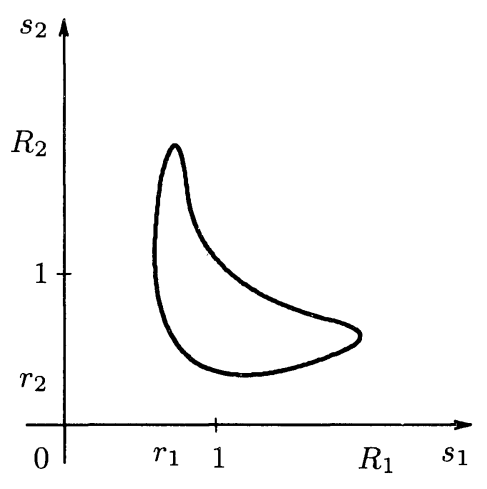

Рис. 2

1.1. Задача о вероятности остановки. Для блуждания $S_{n}$ состояния $\left\{\left(\gamma_{1}, 0\right),\left(0, \gamma_{2}\right): \gamma_{1}, \gamma_{2} \in \mathbf{N}\right\}$ являются поглощающими. Вероятность остановки - вероятность попадания в поглощающее состояние равна:

$$
q_{\left(\gamma_{1}, 0\right)}^{\left(\alpha_{1}, \alpha_{2}\right)}=\lim _{n \rightarrow \infty} P_{\left(\gamma_{1}, 0\right)}^{\left(\alpha_{1}, \alpha_{2}\right)}(n), \quad q_{\left(0, \gamma_{2}\right)}^{\left(\alpha_{1}, \alpha_{2}\right)}=\lim _{n \rightarrow \infty} P_{\left(0, \gamma_{2}\right)}^{\left(\alpha_{1}, \alpha_{2}\right)}(n)
$$

$\left(\alpha_{1}, \alpha_{2}\right) \in \mathbf{N}^{2}$ (из соображений симметрии достаточно иметь выражения для вероятностей $\left.q_{\left(0, \gamma_{2}\right)}^{\left(\alpha_{1}, \alpha_{2}\right)}\right)$. Задача о нахождении вероятности остановки в точке $\left(0, \gamma_{2}\right)$ рассматривалась многими авторами. В [1] выявлены и исследованы алгебраические группы, связанные со случайными блужданиями в четверти плоскости, разработаны аналитические методы уравнений Винера-Хопфа для решения задач о многомерных случайных блужданиях. В докладе [5] дано интегральное представление для $q_{\left(0, \gamma_{2}\right)}^{\left(1, \alpha_{2}\right)}$ в симметричном случае $h\left(s_{1}, s_{2}\right)=p_{01} s_{1}^{2} s_{2}+p_{10} s_{1} s_{2}^{2}+p_{10} s_{1}+p_{01} s_{2}$. При $h\left(s_{1}, s_{2}\right)=p_{22} s_{1}^{2} s_{2}^{2}+p_{21} s_{1}^{2} s_{2}+p_{12} s_{1} s_{2}^{2}+p_{20} s_{1}^{2}+p_{02} s_{2}^{2}+p_{10} s_{1}+p_{01} s_{2}+p_{00}$ (рис. 1 , случай б)) искомое выражение для вероятностей (1) содержит эллиптические функции (см. [1]). В обзоре [3] задача о нахождении вероятности остановки на границе для случайного блуждания $S_{n}$ отнесена к типу 3 б). В.А. Малышев указывает, что разработанная им «техника может быть использована для решения уравнений в частных производных с постоянными коэффициентами в квадранте $\mathbf{R}_{+}^{2} \gg[3$, с. 21]. 
Для задачи типа 3б) дифференциальное уравнение такого вида получено в п. 2 настояшей работы - это уравнение в частных производных (4) с условиями (5) на границах квадранта $\mathbf{R}_{+}^{2}$. Уравнение (4) определяет вид интегрального представления (9) для вероятностей $q_{\left(0, \gamma_{2}\right)}^{\left(\alpha_{1}, \alpha_{2}\right)}$. В теоремах 2-4 даны выражения вида (9) для вероятностей (1) при частных предположениях о функции $h\left(s_{1}, s_{2}\right)$, исходя из полученной в п. 3 системы функциональных уравнений (16), (17). Аналогичным путем из (16), (17) могут быть получены интегральные представления для вероятностей $q_{\left(0, \gamma_{2}\right)}^{\left(\alpha_{1}, \alpha_{2}\right)}$ в случаях $h\left(s_{1}, s_{2}\right)=\sum_{k=0}^{\infty} s_{1}^{k} h_{k}\left(s_{1} s_{2}\right)$, $h\left(s_{1}, s_{2}\right)=\sum_{k=0}^{\infty} s_{2}^{k} h_{k}\left(s_{1} s_{2}\right)$, где $h_{k}(s), k=0,1, \ldots$, - положительные производящие функции. Данная в теореме 5 формула вида (9) для случая $h\left(s_{1}, s_{2}\right)=p_{20} s_{1}^{2}+p_{02} s_{2}^{2}+p_{00}$ получена прямым решением уравнения (4). Ограниченный объем статьи не позволяет привести такой же вывод (см. замечание 3 ) выражений для вероятностей $q_{\left(0, \gamma_{2}\right)}^{\left(\alpha_{1}\right)}$ в случаях $h\left(s_{1}, s_{2}\right)=p_{20} s_{1}^{2}+p_{02} s_{2}^{2}+p_{10} s_{1}+p_{01} s_{2}+p_{00}$ и $h\left(s_{1}, s_{2}\right)=$ $p_{22} s_{1}^{2} s_{2}^{2}+p_{21} s_{1}^{2} s_{2}+p_{12} s_{1} s_{2}^{2}+p_{20} s_{1}^{2}+p_{02} s_{2}^{2}$. Автору работы не удалось получить выражение для вероятностей (1) при произвольной производящей функции $h\left(s_{1}, s_{2}\right)$; совокупность рассмотренных частных случаев позволяет сделать предположение, что такое аналитическое выражение вида (9) существует.

А. А. Могульский и Б. А. Рогозин в недавней работе [6] применили метод факторизации к общему случайному блужданию в квадранте $\mathbf{R}_{+}^{2}$. Найдены, в частности, асимптотические формулы для вероятности выхода случайного блуждания из положительного квадранта, когда стремится к бесконечности время выхода или положение точки выхода. Из результатов [6, ч. I] следуют экспоненциальные представления для рассматриваемых в настоящей работе функций $\varphi_{1}(s), \varphi_{2}(s)$ (см. в $[6$, ч. I] выражения для функций $\left.V_{z+}(\lambda), V_{z-}(\lambda), v(z, \lambda)\right)$.

\section{2. Ветвящийся процесс с двумя типами частиц и одним} комплексом взаимодействия ([4]). Рассмотрим однородный во времени марковский процесс $\mu(t)=\left(\mu_{1}(t), \mu_{2}(t)\right)$ на фазовом пространстве $\mathrm{N}^{2}$ с непрерывным временем $t, t \in[0, \infty)$, и переходными вероятностями

$$
P_{\left(\beta_{1}, \beta_{2}\right)}^{\left(\alpha_{1}, \alpha_{2}\right)}(t)=\mathbf{P}\left\{\mu(t)=\left(\beta_{1}, \beta_{2}\right) \mid \mu(0)=\left(\alpha_{1}, \alpha_{2}\right)\right\}
$$

Пусть при $\Delta t \rightarrow 0$ переходные вероятности имеют вид $(\lambda>0)$

$$
\begin{aligned}
& P_{\left(\alpha_{1}, \alpha_{2}\right)}^{\left(\alpha_{1}, \alpha_{2}\right)}(\Delta t)=1-\alpha_{1} \alpha_{2} \lambda \Delta t+o(\Delta t) \\
& P_{\left(\beta_{1}, \beta_{2}\right)}^{\left(\alpha_{1}, \alpha_{2}\right)}(\Delta t)=p_{\beta_{1}-\alpha_{1}+1, \beta_{2}-\alpha_{2}+1} \alpha_{1} \alpha_{2} \lambda \Delta t+o(\Delta t)
\end{aligned}
$$


если $\alpha_{1} \neq \beta_{1}$ или $\alpha_{2} \neq \beta_{2}$. Введем производящие функции переходных вероятностей $\left(\left|s_{1}\right| \leqslant 1,\left|s_{2}\right| \leqslant 1\right)$

$$
\begin{array}{ll}
F_{\left(\alpha_{1}, \alpha_{2}\right)}\left(t ; s_{1}, s_{2}\right)= & \sum_{\beta_{1}, \beta_{2}=0}^{\infty} P_{\left(\beta_{1}, \beta_{2}\right)}^{\left(\alpha_{1}, \alpha_{2}\right)}(t) s_{1}^{\beta_{1}} s_{2}^{\beta_{2}}, \quad\left(\alpha_{1}, \alpha_{2}\right) \in \mathbf{N}^{2}, \\
G_{\left(\beta_{1}, \beta_{2}\right)}\left(t ; z_{1}, z_{2}\right)= & \sum_{\alpha_{1}, \alpha_{2}=0}^{\infty} \frac{z_{1}^{\alpha_{1}} z_{2}^{\alpha_{2}}}{\alpha_{1} ! \alpha_{2} !} P_{\left(\beta_{1}, \beta_{2}\right)}^{\left(\alpha_{1}, \alpha_{2}\right)}(t),
\end{array}
$$

и дифференциальный оператор

$$
h\left(\frac{\partial}{\partial z_{1}}, \frac{\partial}{\partial z_{2}}\right)=\sum_{\gamma_{1}, \gamma_{2}=0}^{\infty} p_{\gamma_{1} \gamma_{2}} \frac{\partial^{\gamma_{1}+\gamma_{2}}}{\partial z_{1}^{\gamma_{1}} \partial z_{2}^{\gamma_{2}}}
$$

Вторая (прямая) система дифференциальных уравнений Колмогорова для процесса $\mu(t)$ равносильна линейному уравнению в частных производных второго порядка (см. [4])

$$
\frac{\partial F_{\left(\alpha_{1}, \alpha_{2}\right)}\left(t ; s_{1}, s_{2}\right)}{\partial t}=\lambda\left(h\left(s_{1}, s_{2}\right)-s_{1} s_{2}\right) \frac{\partial^{2} F_{\left(\alpha_{1}, \alpha_{2}\right)}\left(t ; s_{1}, s_{2}\right)}{\partial s_{1} \partial s_{2}}
$$

с начальным условием $F_{\left(\alpha_{1}, \alpha_{2}\right)}\left(0 ; s_{1}, s_{2}\right)=s_{1}^{\alpha_{1}} s_{2}^{\alpha_{2}}$. Первая (обратная) система дифференциальных уравнений Колмогорова для процесса $\mu(t)$ равносильна линейному уравнению (см. [4]; ср. [18, теорема 1])

$$
\frac{\partial G_{\left(\beta_{1}, \beta_{2}\right)}\left(t ; z_{1}, z_{2}\right)}{\partial t}=\lambda z_{1} z_{2}\left(h\left(\frac{\partial}{\partial z_{1}}, \frac{\partial}{\partial z_{2}}\right)-\frac{\partial^{2}}{\partial z_{1} \partial z_{2}}\right) G_{\left(\beta_{1}, \beta_{2}\right)}\left(t ; z_{1}, z_{2}\right)
$$

с начальным условием $G_{\left(\beta_{1}, \beta_{2}\right)}\left(0 ; z_{1}, z_{2}\right)=z_{1}^{\beta_{1}} z_{2}^{\beta_{2}} /\left(\beta_{1} ! \beta_{2} !\right)$.

В таком ветвящемся процессе событие $\left\{\mu(t)=\left(\beta_{1}, \beta_{2}\right)\right\}$ интерпретируется как наличие совокупности из $\beta_{1}$ частиц типа $T_{1}$ и $\beta_{2}$ частиц типа $T_{2}$ в момент времени $t$ (особи мужского рода и особи женского рода [4], [8]). Пусть процесс находится в начальном состоянии, соответствующем вектору $\left(\alpha_{1}, \alpha_{2}\right)$. Можно полагать, что через случайное время $\tau_{\left(\alpha_{1}, \alpha_{2}\right)}, \mathbf{P}\left\{\tau_{\left(\alpha_{1}, \alpha_{2}\right)}<t\right\}=1-e^{-\alpha_{1} \alpha_{2} \lambda t}$ происходит взаимодействие частицы типа $T_{1}$ с частицей типа $T_{2}$. Эта пара частиц независимо от других частиц превращается в новую группу из $\gamma_{1}$ частиц типа $T_{1}$ и $\gamma_{2}$ частиц типа $T_{2}: T_{1}+T_{2} \rightarrow \gamma_{1} T_{1}+\gamma_{2} T_{2}$, с распределением вероятностей $\left\{p_{\gamma_{1} \gamma_{2}}\right\}$; процесс переходит в состояние, соответствующее вектору $\left(\alpha_{1}+\gamma_{1}-1, \alpha_{2}+\gamma_{2}-1\right)$. Далее аналогичная эволюция случайного процесса. Для ветвящегося процесса $\mu(t)$ возможно вырождение в одном из поглощающих состояний из множества $\left\{\left(\gamma_{1}, 0\right),\left(0, \gamma_{2}\right): \gamma_{1}, \gamma_{2} \in \mathbf{N}\right\}$ (остались частицы одного типа, взаимодействия $T_{1}+T_{2}$ невозможны). 
2.1. Стационарное первое уравнение для экспоненциальной производящей функции. Из описания процесса $\mu(t)$ следует, что если рассматривать $\mu(t)$ только в моменты изменения состояния, т.е. «вложенную цепь Маркова», то она совпадает с определенным в п. 1 случайным блужданием $S_{n}$. Вероятность вырождения процесса $\mu(t)$ совпадает с вероятностью остановки блуждания $S_{n}$ при одинаковом начальном условии (ср. [2, гл. $2, \S 1$, теорема 1$])$ :

$$
q_{\left(\gamma_{1}, 0\right)}^{\left(\alpha_{1}, \alpha_{2}\right)}=\lim _{t \rightarrow \infty} P_{\left(\gamma_{1}, 0\right)}^{\left(\alpha_{1}, \alpha_{2}\right)}(t), \quad q_{\left(0, \gamma_{2}\right)}^{\left(\alpha_{1}, \alpha_{2}\right)}=\lim _{t \rightarrow \infty} P_{\left(0, \gamma_{2}\right)}^{\left(\alpha_{1}, \alpha_{2}\right)}(t), \quad\left(\alpha_{1}, \alpha_{2}\right) \in \mathbf{N}^{2} .
$$

Для вероятностей $q_{\left(0, \gamma_{2}\right)}^{\left(\alpha_{1}, \alpha_{2}\right)}$ введем экспоненциальную производящую функцию

$$
g_{\left(0, \gamma_{2}\right)}\left(z_{1}, z_{2}\right)=\sum_{\alpha_{1}, \alpha_{2}=0}^{\infty} \frac{z_{1}^{\alpha_{1}} z_{2}^{\alpha_{2}}}{\alpha_{1} ! \alpha_{2} !} q_{\left(0, \gamma_{2}\right)}^{\left(\alpha_{1}, \alpha_{2}\right)}
$$

Функция $g_{\left(0, \gamma_{2}\right)}\left(z_{1}, z_{2}\right)$ - аналитическая по переменным $z_{1}, z_{2}$, так как

$$
\left|g_{\left(0, \gamma_{2}\right)}\left(z_{1}, z_{2}\right)\right| \leqslant \sum_{\alpha_{1}, \alpha_{2}=0}^{\infty} \frac{\left|z_{1}\right|^{\alpha_{1}}\left|z_{2}\right|^{\alpha_{2}}}{\alpha_{1} ! \alpha_{2} !}\left|q_{\left(0, \gamma_{2}\right)}^{\left(\alpha_{1}, \alpha_{2}\right)}\right| \leqslant e^{\left|z_{1}\right|+\left|z_{2}\right|}
$$

Аналогично теореме 2 работы [18] показывается, что $g_{\left(0, \gamma_{2}\right)}\left(z_{1}, z_{2}\right)=$ $\lim _{t \rightarrow \infty} G_{\left(0, \gamma_{2}\right)}\left(t ; z_{1}, z_{2}\right)$ и $g_{\left(0, \gamma_{2}\right)}\left(z_{1}, z_{2}\right)$ удовлетворяет стационарному первому уравнению

$$
\left[h\left(\frac{\partial}{\partial z_{1}}, \frac{\partial}{\partial z_{2}}\right)-\frac{\partial^{2}}{\partial z_{1} \partial z_{2}}\right] g_{\left(0, \gamma_{2}\right)}\left(z_{1}, z_{2}\right)=0
$$

Рассмотрим поглощающие состояния $\left(0, \gamma_{2}\right), \gamma_{2}=1,2, \ldots$ Из очевидных равенств для вероятностей остановки: $q_{\left(0, \gamma_{2}\right)}^{\left(0, \gamma_{2}\right)}=1, q_{\left(0, \gamma_{2}\right)}^{\left(0, \alpha_{2}\right)}=0$, если $\alpha_{2} \neq \gamma_{2} ; q_{\left(0, \gamma_{2}\right)}^{\left(\alpha_{1}, 0\right)}=0$, если $\alpha_{1}=0,1, \ldots$, получаем условия на границах $z_{1}=0, z_{2}=0$ квадранта $\mathbf{R}_{+}^{2}$ :

$$
g_{\left(0, \gamma_{2}\right)}\left(0, z_{2}\right)=\frac{z_{2}^{\gamma_{2}}}{\gamma_{2} !}, \quad g_{\left(0, \gamma_{2}\right)}\left(z_{1}, 0\right)=0
$$

Для поглощающего состояния $(0,0)$ получаем граничные условия $g_{(0,0)}\left(0, z_{2}\right)=1, g_{(0,0)}\left(z_{1}, 0\right)=1$, рассмотрение которых аналогично случаю (5).

2.2. Об интегральном представлении для вероятностей $q_{\left(0, \gamma_{2}\right)}^{\left(\alpha_{1}, \alpha_{2}\right)}$. Ссылки на ряд работ по граничной задаче типа (4), (5) приведены в обзоре [3]. При рассмотрении задачи (4), (5) - нахождении 
явного выражения для функции $g_{\left(0, \gamma_{2}\right)}\left(z_{1}, z_{2}\right)$ - можно использовать некоторые подходы общей теории линейных дифференциальных операторов в частных производных с постоянными коэффициентами [11]. Для уравнения (4) характеристическим является уравнение

$$
h\left(s_{1}, s_{2}\right)-s_{1} s_{2}=0
$$

(cp. [2, гл. 2, §1, уравнение (9)]). Вводим функции $\varphi_{1}\left(s_{2}\right), \varphi_{2}\left(s_{1}\right)$, определяемые характеристическим уравнением $h\left(\varphi_{1}\left(s_{2}\right), s_{2}\right)-\varphi_{1}\left(s_{2}\right) s_{2}=0$; $h\left(s_{1}, \varphi_{2}\left(s_{1}\right)\right)-s_{1} \varphi_{2}\left(s_{1}\right)=0$. «Общее решение» уравнения (4) имеет вид (см. об экспоненциальном представлении решений уравнений в частных производных $[11, \S 2])$

$$
\int_{Q} e^{z_{1} \varphi_{1}\left(s_{2}\right)+z_{2} s_{2}} \mu^{1}\left(d s_{2}\right)=\int_{Q} e^{z_{1} s_{1}+z_{2} \varphi_{2}\left(s_{1}\right)} \mu^{2}\left(d s_{1}\right)
$$

где $\mu^{1}\left(d s_{2}\right), \mu^{2}\left(d s_{1}\right)$ - меры, сосредоточенные на множестве $Q=$ $\left\{\left(s_{1}, s_{2}\right): h\left(s_{1}, s_{2}\right)-s_{1} s_{2}=0\right\}$. Действительно, подставим, например, первое из выражений (7) в уравнение (4), тогда получаем после формальных вычислений:

$$
\begin{aligned}
& {\left[h\left(\frac{\partial}{\partial z_{1}}, \frac{\partial}{\partial z_{2}}\right)-\frac{\partial^{2}}{\partial z_{1} \partial z_{2}}\right] \int_{Q} e^{z_{1} \varphi_{1}\left(s_{2}\right)+z_{2} s_{2}} \mu^{1}\left(d s_{2}\right)} \\
& \quad=\int_{Q}\left[h\left(\frac{\partial}{\partial z_{1}}, \frac{\partial}{\partial z_{2}}\right)-\frac{\partial^{2}}{\partial z_{1} \partial z_{2}}\right] e^{z_{1} \varphi_{1}\left(s_{2}\right)+z_{2} s_{2}} \mu^{1}\left(d s_{2}\right) \\
& \quad=\int_{Q} e^{z_{1} \varphi_{1}\left(s_{2}\right)+z_{2} s_{2}}\left(h\left(\varphi_{1}\left(s_{2}\right), s_{2}\right)-\varphi_{1}\left(s_{2}\right) s_{2}\right) \mu^{1}\left(d s_{2}\right)=0 .
\end{aligned}
$$

Из (7), разложения экспоненты

$$
e^{z_{1} s_{1}+z_{2} s_{2}}=\sum_{\alpha_{1}, \alpha_{2}=0}^{\infty} \frac{z_{1}^{\alpha_{1}} z_{2}^{\alpha_{2}}}{\alpha_{1} ! \alpha_{2} !} s_{1}^{\alpha_{1}} s_{2}^{\alpha_{2}}
$$

и определения (2) производящей функции $g_{\left(0, \gamma_{2}\right)}\left(z_{1}, z_{2}\right)$, учитывая, что меры в (7) зависят от граничных условий (5), получаем, что искомое выражение для вероятности остановки в точке $\left(0, \gamma_{2}\right)$ имеет вид

$$
q_{\left(0, \gamma_{2}\right)}^{\left(\alpha_{1}, \alpha_{2}\right)}=\int_{Q} \varphi_{1}^{\alpha_{1}}\left(s_{2}\right) s_{2}^{\alpha_{2}} \mu_{\left(0, \gamma_{2}\right)}^{1}\left(d s_{2}\right)=\int_{Q} s_{1}^{\alpha_{1}} \varphi_{2}^{\alpha_{2}}\left(s_{1}\right) \mu_{\left(0, \gamma_{2}\right)}^{2}\left(d s_{1}\right) .
$$

3. Система функциональных уравнений для производящих функций вероятностей остановки. Введем производящие функции

$$
\Phi_{1}^{\left(\alpha_{1}, \alpha_{2}\right)}(s)=\sum_{\gamma_{1}=0}^{\infty} q_{\left(\gamma_{1}, 0\right)}^{\left(\alpha_{1}, \alpha_{2}\right)} s^{\gamma_{1}}, \quad \Phi_{2}^{\left(\alpha_{1}, \alpha_{2}\right)}(s)=\sum_{\gamma_{2}=0}^{\infty} q_{\left(0, \gamma_{2}\right)}^{\left(\alpha_{1}, \alpha_{2}\right)} s^{\gamma_{2}},
$$

$\left(\alpha_{1}, \alpha_{2}\right) \in \mathbf{N}^{2}$ (аналитические в круге $|s| \leqslant 1$ ). Для вывода системы уравнений для $\Phi_{1}^{\left(\alpha_{1}, \alpha_{2}\right)}(s), \Phi_{2}^{\left(\alpha_{1}, \alpha_{2}\right)}(s)$ потребуются вспомогательные определения. 


\section{1. Случайное блуждание $\widehat{S}_{n}$ в правой полуплоскости.} Случайное блуждание $\widetilde{S}_{n}$ в верхней полуплоскости. Определим случайное блуждание $\widehat{S}_{n}, n=0,1, \ldots$, на множестве $\mathbf{N} \times \mathbf{Z}=$ $\left\{\left(\alpha_{1}, \alpha_{2}\right): \alpha_{1} \in \mathbf{N}, \alpha_{2} \in \mathbf{Z}\right\}$, где $\mathbf{Z}=\{\ldots,-1,0,1, \ldots\}$. Переходные вероятности за $n$ шагов обозначим $\widehat{P}_{\left(\beta_{1}, \beta_{2}\right)}^{\left(\alpha_{1}, \alpha_{2}\right)}(n)$. Переходные вероятности за один шаг полагаем равными: $\mathbf{P}\left\{\widehat{S}_{n+1}=\left(\beta_{1}, \beta_{2}\right) \mid \widehat{S}_{n}=\left(\alpha_{1}, \alpha_{2}\right)\right\}=$ $p_{\beta_{1}-\alpha_{1}+1, \beta_{2}-\alpha_{2}+1}$, если $\alpha_{1}>0$ и $\beta_{1}-\alpha_{1}+1 \geqslant 0, \beta_{2}-\alpha_{2}+1 \geqslant 0$; $\mathbf{P}\left\{\widehat{S}_{n+1}=\left(\alpha_{1}, \alpha_{2}\right) \mid \widehat{S}_{n}=\left(\alpha_{1}, \alpha_{2}\right)\right\}=1$, если $\alpha_{1}=0$. Состояния $\left\{\left(0, \gamma_{2}\right): \gamma_{2} \in \mathbf{Z}\right\}$ являются поглощающими для блуждания $\widehat{S}_{n}$. Существуют пределы $\widehat{q}_{\left(0, \gamma_{2}\right)}^{\left(\alpha_{1}, \alpha_{2}\right)}=\lim _{n \rightarrow \infty} \widehat{P}_{\left(0, \gamma_{2}\right)}^{\left(\alpha_{1}, \alpha_{2}\right)}(n),\left(\alpha_{1}, \alpha_{2}\right) \in \mathbf{N} \times \mathbf{Z}, \gamma_{2} \in \mathbf{Z}$ (вероятность остановки в точке границы $\left(0, \gamma_{2}\right)$ ).

Аналогично определим случайное блуждание $\widetilde{S}_{n}, n=0,1, \ldots$, на множестве $\mathbf{Z} \times \mathbf{N}=\left\{\left(\alpha_{1}, \alpha_{2}\right): \alpha_{1} \in \mathbf{Z}, \alpha_{2} \in \mathbf{N}\right\}$. Переходные вероятности за $n$ шагов обозначим $\widetilde{P}_{\left(\beta_{1}, \beta_{2}\right)}^{\left(\alpha_{1}, \alpha_{2}\right)}(n)$. Переходные вероятности за один шаг полагаем равными: $\mathbf{P}\left\{\widetilde{S}_{n+1}=\left(\beta_{1}, \beta_{2}\right) \mid \widetilde{S}_{n}=\left(\alpha_{1}, \alpha_{2}\right)\right\}=$ $p_{\beta_{1}-\alpha_{1}+1, \beta_{2}-\alpha_{2}+1}$, если $\alpha_{2}>0$ и $\beta_{1}-\alpha_{1}+1 \geqslant 0, \beta_{2}-\alpha_{2}+1 \geqslant 0$; $\mathbf{P}\left\{\widetilde{S}_{n+1}=\left(\alpha_{1}, \alpha_{2}\right) \mid \widetilde{S}_{n}=\left(\alpha_{1}, \alpha_{2}\right)\right\}=1$, если $\alpha_{2}=0$. Состояния $\left\{\left(\gamma_{1}, 0\right): \gamma_{1} \in \mathbf{Z}\right\}$ являются поглощающими для блуждания $\widetilde{S}_{n}$. Существуют пределы $\widetilde{q}_{\left(\gamma_{1}, 0\right)}^{\left(\alpha_{1}, \alpha_{2}\right)}=\lim _{n \rightarrow \infty} \widetilde{P}_{\left(\gamma_{1}, 0\right)}^{\left(\alpha_{1}, \alpha_{2}\right)}(n),\left(\alpha_{1}, \alpha_{2}\right) \in \mathbf{Z} \times \mathbf{N}, \gamma_{1} \in \mathbf{Z}$. Введем функции:

$$
\begin{aligned}
& \varphi_{1}^{\left(\alpha_{1}, \alpha_{2}\right)}(s)=\sum_{\gamma_{2}=-\infty}^{\infty} \widehat{q}_{\left(0, \gamma_{2}\right)}^{\left(\alpha_{1}, \alpha_{2}\right)} s^{\gamma_{2}}, \\
& \left(\alpha_{1}, \alpha_{2}\right) \in \mathbf{N} \times \mathbf{Z}, \quad \varphi_{1}(s)=\varphi_{1}^{(1,0)}(s) ; \\
& \varphi_{2}^{\left(\alpha_{1}, \alpha_{2}\right)}(s)=\sum_{\gamma_{1}=-\infty}^{\infty} \widetilde{q}_{\left(\gamma_{1}, 0\right)}^{\left(\alpha_{1}, \alpha_{2}\right)} s^{\gamma_{1}}, \\
& \left(\alpha_{1}, \alpha_{2}\right) \in \mathbf{Z} \times \mathbf{N}, \quad \varphi_{2}(s)=\varphi_{2}^{(0,1)}(s) .
\end{aligned}
$$

Ряды (10), (11) представляют собой ряды Лорана [9]. Ряд $\varphi_{1}(s)$ сходится в кольце $r_{2}<|s|<R_{2}$, ряд $\varphi_{2}(s)$ сходится в кольце $r_{1}<|s|<R_{1}$; заметим, что $r_{i} \leqslant 1 \leqslant R_{i}, i=1,2$.

3.2. Вывод системы уравнений. Для простоты мы ограничиваемся дальше случаем, когда производящая функция $h\left(s_{1}, s_{2}\right)$ аналитична по своим аргументам при всех $s_{1}, s_{2}$.

Лемма 1. Справедливы соотношения для производящих функиий:

$$
\begin{array}{ll}
\varphi_{1}^{\left(\alpha_{1}, \alpha_{2}\right)}(s)=\varphi_{1}^{\alpha_{1}}(s) s^{\alpha_{2}}, & \left(\alpha_{1}, \alpha_{2}\right) \in \mathbf{N} \times \mathbf{Z} \\
h\left(\varphi_{1}(s), s\right)-\varphi_{1}(s) s=0, & r_{2}<|s|<R_{2} \\
\varphi_{2}^{\left(\alpha_{1}, \alpha_{2}\right)}(s)=s^{\alpha_{1}} \varphi_{2}^{\alpha_{2}}(s), & \left(\alpha_{1}, \alpha_{2}\right) \in \mathbf{Z} \times \mathbf{N} \\
h\left(s, \varphi_{2}(s)\right)-s \varphi_{2}(s)=0, & r_{1}<|s|<R_{1} .
\end{array}
$$


Д о к а 3 а т е л ь с т в о. Получим соотношения (12) и (13); соотношения (14) и (15) выводятся аналогично. Для случайного блуждания $\widehat{S}_{n}$ очевидно равенство $\widehat{q}_{\left(0, \gamma_{2}\right)}^{\left(\alpha_{1}, \alpha_{2}\right)}=\widehat{q}_{\left(0, \gamma_{2}-\alpha_{2}\right)}^{\left(\alpha_{1}, 0\right)},\left(\alpha_{1}, \alpha_{2}\right) \in \mathbf{N} \times \mathbf{Z}, \gamma_{2} \in \mathbf{Z}$. Отсюда и из определения (10) получаем (здесь и далее $\left.r_{2}<|s|<R_{2}\right) \varphi_{1}^{\left(\alpha_{1}, \alpha_{2}\right)}(s)=$ $\varphi_{1}^{\left(\alpha_{1}, 0\right)}(s) s^{\alpha_{2}},\left(\alpha_{1}, \alpha_{2}\right) \in \mathbf{N} \times \mathbf{Z}$. В силу строго марковского свойства процесса $\widehat{S}_{n}, n=0,1, \ldots$, имеем равенство $\widehat{q}_{\left(0, \gamma_{2}\right)}^{\left(\alpha_{1}, 0\right)}=\sum_{l=-\infty}^{\infty} \widehat{q}_{(0, l)}^{(1,0)} \widehat{q}_{\left(0, \gamma_{2}\right)}^{\left(\alpha_{1}-1, l\right)}$, $\alpha_{1}=1,2, \ldots, \gamma_{2} \in \mathbf{Z}$. Свертка с помощью функции (10) дает (перестановка знаков суммирования законна) $\varphi_{1}^{\left(\alpha_{1}, 0\right)}(s)=\varphi_{1}(s) \varphi_{1}^{\left(\alpha_{1}-1,0\right)}(s)$, $\alpha_{1}=1,2, \ldots$, и, следовательно,

$$
\varphi_{1}^{\left(\alpha_{1}, 0\right)}(s)=\varphi_{1}^{\alpha_{1}}(s), \quad \alpha_{1} \in \mathbf{N} .
$$

Соотношение (12) доказано. Соотношение (13) также следует из строго марковского свойства $\widehat{S}_{n}: \varphi_{1}(s) s=\varphi_{1}^{(1,1)}(s)=\sum_{\alpha_{1}, \alpha_{2}=0}^{\infty} p_{\alpha_{1} \alpha_{2}} \varphi_{1}^{\left(\alpha_{1}, \alpha_{2}\right)}(s)=$ $\sum_{\alpha_{1}, \alpha_{2}=0}^{\infty} p_{\alpha_{1} \alpha_{2}} \varphi_{1}^{\alpha_{1}}(s) s^{\alpha_{2}}=h\left(\varphi_{1}(s), s\right)$. Лемма 1 доказана.

Теорема 1. Справедливы соотношения для производящих функиий:

$$
\begin{gathered}
\Phi_{1}^{\left(\alpha_{1}, \alpha_{2}\right)}(s)=s^{\alpha_{1}} \varphi_{2}^{\alpha_{2}}(s)-\Phi_{2}^{\left(\alpha_{1}, \alpha_{2}\right)}\left(\varphi_{2}(s)\right)+\Phi_{2}^{\left(\alpha_{1}, \alpha_{2}\right)}(0) \\
r_{1}<|s|<R_{1} \\
\Phi_{2}^{\left(\alpha_{1}, \alpha_{2}\right)}(s)=\varphi_{1}^{\alpha_{1}}(s) s^{\alpha_{2}}-\Phi_{1}^{\left(\alpha_{1}, \alpha_{2}\right)}\left(\varphi_{1}(s)\right)+\Phi_{1}^{\left(\alpha_{1}, \alpha_{2}\right)}(0) \\
r_{2}<|s|<R_{2} .
\end{gathered}
$$

Д о к а з а т е л ь с т в о. Получим соотношение (17); соотношение (16) выводится аналогично. Учитывая строго марковское свойство процессов $S_{n}, \widehat{S}_{n}$, имеем равенства:

$$
\begin{aligned}
& q_{\left(0, \gamma_{2}\right)}^{\left(\alpha_{1}, \alpha_{2}\right)}=\widehat{q}_{\left(0, \gamma_{2}\right)}^{\left(\alpha_{1}, \alpha_{2}\right)}-\sum_{\gamma_{1}=1}^{\infty} q_{\left(\gamma_{1}, 0\right)}^{\left(\alpha_{1}, \alpha_{2}\right)} \widehat{q}_{\left(0, \gamma_{2}\right)}^{\left(\gamma_{1}, 0\right)}, \quad\left(\alpha_{1}, \alpha_{2}\right) \in \mathbf{N}^{2}, \quad \gamma_{2} \in \mathbf{N} ; \\
& \widehat{q}_{\left(0, \gamma_{2}\right)}^{\left(\alpha_{1}, \alpha_{2}\right)}=\sum_{\gamma_{1}=1}^{\infty} q_{\left(\gamma_{1}, 0\right)}^{\left(\alpha_{1}, \alpha_{2}\right)} \widehat{q}_{\left(0, \gamma_{2}\right)}^{\left(\gamma_{1}, 0\right)}, \quad\left(\alpha_{1}, \alpha_{2}\right) \in \mathbf{N}^{2}, \quad \gamma_{2}=-1,-2, \ldots .
\end{aligned}
$$

Свертывая первое равенство, получаем $\left(|s|<R_{2}\right.$; рассматриваемые ряды сходятся абсолютно, законна перестановка знаков суммирования)

$$
\sum_{\gamma_{2}=0}^{\infty} q_{\left(0, \gamma_{2}\right)}^{\left(\alpha_{1}, \alpha_{2}\right)} s^{\gamma_{2}}=\sum_{\gamma_{2}=0}^{\infty} \hat{q}_{\left(0, \gamma_{2}\right)}^{\left(\alpha_{1}, \alpha_{2}\right)} s^{\gamma_{2}}-\sum_{\gamma_{1}=1}^{\infty} q_{\left(\gamma_{1}, 0\right)}^{\left(\alpha_{1}, \alpha_{2}\right)} \sum_{\gamma_{2}=0}^{\infty} \hat{q}_{\left(0, \gamma_{2}\right)}^{\left(\gamma_{1}, 0\right)} s^{\gamma_{2}}
$$

Далее, из второго равенства:

$$
0=\sum_{\gamma_{2}=-1}^{-\infty} \widehat{q}_{\left(0, \gamma_{2}\right)}^{\left(\alpha_{1}, \alpha_{2}\right)} s^{\gamma_{2}}-\sum_{\gamma_{1}=1}^{\infty} q_{\left(\gamma_{1}, 0\right)}^{\left(\alpha_{1}, \alpha_{2}\right)} \sum_{\gamma_{2}=-1}^{-\infty} \widehat{q}_{\left(0, \gamma_{2}\right)}^{\left(\gamma_{1}, 0\right)} s^{\gamma_{2}}, \quad|s|>r_{2} .
$$


Складывая полученные выражения и учитывая (12), получаем

$$
\begin{aligned}
& \sum_{\gamma_{2}=0}^{\infty} q_{\left(0, \gamma_{2}\right)}^{\left(\alpha_{1}, \alpha_{2}\right)} s^{\gamma_{2}}=\varphi_{1}^{\left(\alpha_{1}, \alpha_{2}\right)}(s)-\sum_{\gamma_{1}=1}^{\infty} q_{\left(\gamma_{1}, 0\right)}^{\left(\alpha_{1}, \alpha_{2}\right)} \varphi_{1}^{\left(\gamma_{1}, 0\right)}(s) \\
& \quad=\varphi_{1}^{\alpha_{1}}(s) s^{\alpha_{2}}-\sum_{\gamma_{1}=0}^{\infty} q_{\left(\gamma_{1}, 0\right)}^{\left(\alpha_{1}, \alpha_{2}\right)} \varphi_{1}^{\gamma_{1}}(s)+q_{(0,0)}^{\left(\alpha_{1}, \alpha_{2}\right)}, \quad r_{2}<|s|<R_{2},
\end{aligned}
$$

что совпадает с соотношением (17). Теорема 1 доказана.

Из равенства $(18)$ следует, что функции $\Phi_{i}^{\left(\alpha_{1}, \alpha_{2}\right)}(s)$ являются аналитическими в круге $|s|<R_{i}\left(i=1,2 ;\left(\alpha_{1}, \alpha_{2}\right) \in \mathbf{N}^{2}\right)$.

4. Анализ характеристического уравнения $h\left(s_{1}, s_{2}\right)-s_{1} s_{2}=0$. Рассмотрим определенные в $(10),(11)$ функции $\varphi_{1}(s), \varphi_{2}(s)$ и множество $Q^{+}=\left\{\left(s_{1}, s_{2}\right): h\left(s_{1}, s_{2}\right)-s_{1} s_{2}=0, s_{1}>0, s_{2}>0\right\}$.

Обозначим через $S$ множество всех точек плоскости с целочисленными координатами $\left(\gamma_{1}, \gamma_{2}\right)$, для которых $p_{\gamma_{1}+1, \gamma_{2}+1}>0$ или $\gamma_{1}=\gamma_{2}=0$. Обозначим $S_{L}$ такую решетку целочисленных точек плоскости, которая содержит множество $S$ и не содержит никакой подрешетки, удовлетворяющей этому же свойству. Координаты всех точек решетки $S_{L}$ можно получить, составляя всевозможные линейные комбинации с целыми коэффициентами из координат точек множества $S$ (см. $[2$, гл. $5, \S 4])$. Обозначим $S_{L}^{+}$множество всех точек, которые можно получить, составляя всевозможные линейные комбинации с целыми неотрицательными коэффициентами из координат точек множества $S$. Будем говорить, что для распределения вероятностей $\left\{p_{\gamma_{1} \gamma_{2}},\left(\gamma_{1}, \gamma_{2}\right) \in \mathbf{N}^{2}\right\}$ выполняется условие $\mathrm{A}$, если $S_{L}$ совпадает с $S_{L}^{+}$и решетка $S_{L}$ двумерна. При условии А решетка $S_{L}$ характеризует множество состояний, достижимых для случайного процесса $\mu(t)$ из начального состояния $\left(\alpha_{1}, \alpha_{2}\right)$ : если $\left(\beta_{1}, \beta_{2}\right) \in S_{L}$ и $\alpha_{1}+\beta_{1}+1 \geqslant 0, \alpha_{2}+\beta_{2}+1 \geqslant 0$, то $P_{\left(\alpha_{1}+\beta_{1}+1, \alpha_{2}+\beta_{2}+1\right)}^{\left(\alpha_{1}, \alpha_{2}\right)}\left(t_{0}\right)>0$ при некотором $t_{0}<\infty$; если $\left(\beta_{1}, \beta_{2}\right) \notin S_{L}$, то $P_{\left(\alpha_{1}+\beta_{1}+1, \alpha_{2}+\beta_{2}+1\right)}^{\left(\alpha_{1}, \alpha_{2}\right)}(t) \equiv 0$.

Лемма 2. Пусть для распределения вероятностей $\left\{p_{\gamma_{1} \gamma_{2}},\left(\gamma_{1}, \gamma_{2}\right) \in\right.$ $\left.\mathrm{N}^{2}\right\}$ выполнено условие А. Тогда: $0<r_{2} \leqslant 1 \leqslant R_{2}<\infty, 0<\varphi_{1}\left(r_{2}\right)<\infty$, $0<\varphi_{1}\left(R_{2}\right)<\infty ; 0<r_{1} \leqslant 1 \leqslant R_{1}<\infty, 0<\varphi_{2}\left(r_{1}\right)<\infty, 0<\varphi_{2}\left(R_{1}\right)<\infty$.

Д о к а з а т е л с с в о. Получим утверждение леммы для функции $\varphi_{1}(s)$; функция $\varphi_{2}(s)$ рассматривается аналогично. Из условия $\mathrm{A}$ следует, что разложение (10) для функции $\varphi_{1}(s)$ содержит главную и правильную части [9]. Из (10) следует, что $\varphi_{1}(s)>0$ при $r_{2}<s<R_{2}$.

Рассмотрим $r_{2}$ и $\varphi_{1}\left(r_{2}\right)$.

А). Пусть $p_{\gamma_{1} 0}=0$ при всех $\gamma_{1} \geqslant 1$. По условию А $p_{00}>0$ и существует точка $\left(\beta_{1}, \beta_{2}\right)$ такая, что $0<\beta_{2}<\beta_{1}, p_{\beta_{1} \beta_{2}}>0$; из равенства (13) получаем, что $\varphi_{1}(s) s \geqslant p_{\beta_{1} \beta_{2}} \varphi_{1}^{\beta_{1}}(s) s^{\beta_{2}}$, или $1 \geqslant p_{\beta_{1} \beta_{2}} \varphi_{1}^{\beta_{1}-1}(s) s^{\beta_{2}-1}$ (здесь и далее рассматривается интервал $r_{2}<s<R_{2}$ ). Если $\beta_{2}=1$, то 
$\varphi_{1}(s) \leqslant p_{\beta_{1} \beta_{2}}^{1 /\left(\beta_{1}-1\right)}\left(\beta_{1}>1\right)$ и, полагая в последнем неравенстве $s \downarrow r_{2}$, получаем, что $\varphi_{1}\left(r_{2}\right)$ конечна. При предположении $r_{2}=0$ у функции $\varphi_{1}(s)$ отсутствует главная часть в разложении (10), что противоречит условию А. Если $\beta_{2}>1$, то используем аналогичным образом неравенство $1 \geqslant p_{\beta_{1} \beta_{2}} \varphi_{1}^{\beta_{1}-\beta_{2}}(s)\left(\varphi_{1}(s) s\right)^{\beta_{2}-1} \geqslant p_{\beta_{1} \beta_{2}} \varphi_{1}^{\beta_{1}-\beta_{2}}(s) p_{00}^{\beta_{2}-1}$, где $\beta_{1}-\beta_{2}>0$.

Б). Пусть найдется точка $\left(\gamma_{1}, 0\right)$ такая, что $\gamma_{1} \geqslant 1$ и $p_{\gamma_{1} 0}>0$; из (13) получаем, что $s \geqslant p_{\gamma_{1} 0} \varphi_{1}^{\gamma_{1}-1}(s)$. Если $\gamma_{1}>1$, то из последнего неравенства следует, что $\varphi_{1}\left(r_{2}\right)<\infty$, а отсюда и $r_{2}>0$. Если $\gamma_{1}=1$, то $s \geqslant p_{\gamma_{1} 0}$ и $r_{2}>0$. По условию А существует точка $\left(\beta_{1}, \beta_{2}\right)$ такая, что $\beta_{1}>1$, $p_{\beta_{1} \beta_{2}}>0$; из (13) получаем, что $s \geqslant p_{\beta_{1} \beta_{2}} \varphi_{1}^{\beta_{1}-1}(s) s^{\beta_{2}}$, и, полагая $s \downarrow r_{2}$, делаем вывод, что $\varphi_{1}\left(r_{2}\right)$ конечна.

Рассмотрим $R_{2}$ и $\varphi_{1}\left(R_{2}\right)$.

A). Пусть найдется точка $\left(\gamma_{1}, \gamma_{2}\right)$ такая, что $\gamma_{1} \geqslant 1, \gamma_{2} \geqslant 1$ и $p_{\gamma_{1} \gamma_{2}}>0$ (причем либо $\gamma_{1}>1$, либо $\gamma_{2}>1$, так как $p_{11}=0$ ); из (13) получаем $1 \geqslant p_{\gamma_{1} \gamma_{2}} \varphi_{1}^{\gamma_{1}-1}(s) s^{\gamma_{2}-1}$. Если $\gamma_{1}>1, \gamma_{2}=1$, то $1 \geqslant p_{\gamma_{1} \gamma_{2}} \varphi_{1}^{\gamma_{1}-1}(s)$. При предположении $R_{2}=\infty$ получаем $\overline{\lim }_{s \rightarrow \infty} \varphi_{1}(s)<\infty$, т.е. у функции $\varphi_{1}(s)$ отсутствует правильная часть в разложении (10), что противоречит условию А. Если $\gamma_{1}=1, \gamma_{2}>1$, то $1 \geqslant p_{\gamma_{1} \gamma_{2}} s^{\gamma_{2}-1}$, отсюда $R_{2}<\infty$. Из условия А следует существование точки $\left(\beta_{1}, \beta_{2}\right)$ такой, что $\beta_{1}>1, p_{\beta_{1} \beta_{2}}>0$; из неравенства $s \geqslant p_{\beta_{1} \beta_{2}} \varphi_{1}^{\beta_{1}-1}(s) s^{\beta_{2}}$, полагая $s \uparrow R_{2}$, получаем, что $\varphi_{1}\left(R_{2}\right)$ конечна. Если $\gamma_{1}>1$ и $\gamma_{2}>1$, то далее аналогичные рассуждения.

Б). Пусть $p_{\gamma_{1} \gamma_{2}}=0$ при всех $\gamma_{1} \geqslant 1, \gamma_{2} \geqslant 1$. По условию А существуют точки $\left(\gamma_{1}, 0\right),\left(0, \gamma_{2}\right)$ такие, что $\gamma_{1} \geqslant 2, p_{\gamma_{1} 0}>0$ и $\gamma_{2} \geqslant 2, p_{0 \gamma_{2}}>0$, причем либо $\gamma_{1}>2$, либо $\gamma_{2}>2$; из (13) получаем $s \geqslant p_{\gamma_{1} 0} \varphi_{1}^{\gamma_{1}-1}(s)$, и, соответственно, $\varphi_{1}(s) \geqslant p_{0 \gamma_{2}} s^{\gamma_{2}-1}$. Если $\gamma_{1}>2$, то $s \geqslant p_{\gamma_{1} 0}\left(p_{0 \gamma_{2}} s^{\gamma_{2}-1}\right)^{\gamma_{1}-1}$, и так как $\left(\gamma_{1}-1\right)\left(\gamma_{2}-1\right)>1$, то $R_{2}<\infty$, а отсюда и $\varphi_{1}\left(R_{2}\right)<\infty$. Если $\gamma_{2}>2$, то $\varphi_{1}(s) \geqslant p_{0 \gamma_{2}}\left(p_{\gamma_{1} 0} \varphi_{1}^{\gamma_{1}-1}(s)\right)^{\gamma_{2}-1}$ и далее аналогичные рассуждения. Лемма 2 доказана.

Поскольку ряд $(10)$ для $\varphi_{1}(s)$ имеет неотрицательные коэффициенты, функция $\varphi_{1}(s)$ выпукла вниз на интервале $\left(r_{2}, R_{2}\right)$, а точки $s=r_{2}$, $s=R_{2}$ - особые для функции $\varphi_{1}(s)$. Так же, как в $[2$, гл. $5, \S 4]$, показывается, что эти точки при выполнении условия А являются точками ветвления второго порядка. Если $h\left(s_{1}, s_{2}\right)$ - многочлен, то из теоремы Пелла [18] следует, что множество $Q^{+}$и прямая $s_{1}=C$ (или прямая $s_{2}=C$ ) при любом $C$ имеют не более двух общих точек. Тогда $r_{1}<\varphi_{1}\left(r_{2}\right)<R_{1}, r_{1}<\varphi_{1}\left(R_{2}\right)<R_{1} ; r_{2}<\varphi_{2}\left(r_{1}\right)<R_{2}, r_{2}<\varphi_{2}\left(R_{1}\right)<R_{2}$. На рис. 2 (см. с. 453 ) изображено множество $Q^{+}$при выполнении сформулированных выше условий. Дальнейшее исследование множества $Q^{+}$ связано с рассмотрением параметров критичности [2]

$$
a_{1}=\left.\frac{\partial h\left(s_{1}, s_{2}\right)}{\partial s_{1}}\right|_{s_{1}=1, s_{2}=1}-1, \quad a_{2}=\left.\frac{\partial h\left(s_{1}, s_{2}\right)}{\partial s_{2}}\right|_{s_{1}=1, s_{2}=1}-1 .
$$


5. Интегральное представление для вероятностей $q_{\left(0, \alpha_{2}\right)}^{\left(\alpha_{1}, \alpha_{2}\right)}$ в случае $h\left(s_{1}, s_{2}\right)=s_{2} h_{1}\left(s_{1}, s_{2}\right)$. Положим $h\left(s_{1}, s_{2}\right)=s_{2} h_{1}\left(s_{1}, s_{2}\right)$, где $h_{1}\left(s_{1}, s_{2}\right)$ - вероятностная производящая функция.

Лемма 3 (см. [2]). Пусть $h\left(s_{1}, s_{2}\right)=s_{2} h_{1}\left(s_{1}, s_{2}\right)$, причем для распределения $\left\{p_{\gamma_{1} \gamma_{2}},\left(\gamma_{1}, \gamma_{2}\right) \in \mathbf{N}^{2}\right\}$ имеются вероятности $p_{0 \gamma_{2}}>0, p_{\gamma_{1} \gamma_{2}}>$ 0 с $\gamma_{1} \geqslant 2$ и $p_{\gamma_{1} \gamma_{2}}>0$ c $\gamma_{2}>1$. Тогда функиия $\varphi_{1}(s)$ является аналитической в круге $|s|<R_{2}, 1 \leqslant R_{2}<\infty$, u $0<\varphi_{1}\left(R_{2}\right)<\infty$.

При условиях леммы 3 решетка $S_{L}$ двумерна.

Теорема 2 (см. [2]). Пусть $h\left(s_{1}, s_{2}\right)=s_{2} h_{1}\left(s_{1}, s_{2}\right)$ u вьлполненьл условия леммы 3. Вероятность остановки равна $\left(\alpha_{1} \in \mathbf{N}, \alpha_{2}=1,2, \ldots\right)$

$$
q_{\left(0, \gamma_{2}\right)}^{\left(\alpha_{1}, \alpha_{2}\right)}=\frac{1}{2 \pi i} \oint_{0^{+}} \varphi_{1}^{\alpha_{1}}(s) s^{\alpha_{2}} \frac{d s}{s^{\gamma_{2}+1}}, \quad \gamma_{2} \in \mathbf{N}
$$

Д о к а з а т е л с с в о. Из определения функции $\Phi_{2}^{\left(\alpha_{1}, \alpha_{2}\right)}(s)$ следует, что вероятность остановки выражается через интеграл

$$
q_{\left(0, \gamma_{2}\right)}^{\left(\alpha_{1}, \alpha_{2}\right)}=\frac{1}{2 \pi i} \int_{\theta_{\rho}^{+}} \Phi_{2}^{\left(\alpha_{1}, \dot{\left.\alpha_{2}\right)}\right.}(s) \frac{d s}{s^{\gamma_{2}+1}}, \quad\left(\alpha_{1}, \alpha_{2}\right) \in \mathbf{N}^{2}, \quad \gamma_{2} \in \mathbf{N}
$$

где контур интегрирования - окружность $\theta_{\rho}$ : $|s|=\rho, 0<\rho<R_{2}$. При условии $h\left(s_{1}, s_{2}\right)=s_{2} h_{1}\left(s_{1}, s_{2}\right)$ на скачки случайного блуждания $S_{n}$ имеем $q_{\left(\gamma_{1}, 0\right)}^{\left(\alpha_{1}, \alpha_{2}\right)}=0$, следовательно, $\Phi_{1}^{\left(\alpha_{1}, \alpha_{2}\right)}(s) \equiv 0\left(\alpha_{2}=1,2, \ldots\right)$, и из соотношения (17) получаем, что $\Phi_{2}^{\left(\alpha_{1}, \alpha_{2}\right)}(s)=\varphi_{1}^{\alpha_{1}}(s) s^{\alpha_{2}}$. Подставляя последнее выражение в (20) и учитывая аналитичность функции $\varphi_{1}(s)$ в круге $|s|<R_{2}$, получаем (19). Теорема 2 доказана.

3 а м е ч а н и е 1. Уравнение (4) имеет вид

$$
\left[h_{1}\left(\frac{\partial}{\partial z_{1}}, \frac{\partial}{\partial z_{2}}\right)-\frac{\partial}{\partial z_{1}}\right] g_{\left(0, \gamma_{2}\right)}\left(z_{1}, z_{2}\right)=0 \text {. }
$$

Характеристическое уравнение $h_{1}\left(s_{1}, s_{2}\right)-s_{1}=0$ и функция $\varphi_{1}(s)$ исследованы в $[2$, гл. $5, \S 4]$. Уравнение $(21)$ совпадает со стационарным первым уравнением Колмогорова для ветвящегося процесса (с независимыми частицами) с одним финальным типом $T_{2}$ и одним нефинальным типом $T_{1}$ частиц ([2]). Прямое решение уравнения (21) содержится в работе [19] (случай $k=1$ ).

6. Интегральное представление для вероятностей $q_{\left(0, \gamma_{2}\right)}^{\left(\alpha_{1}, \alpha_{2}\right)}$ в случае $h\left(s_{1}, s_{2}\right)=h_{0}\left(s_{1}\right)+s_{2} h_{1}\left(s_{1}\right)$. Положим $h\left(s_{1}, s_{2}\right)=h_{0}\left(s_{1}\right)+s_{2} h_{1}\left(s_{1}\right)$, где $h_{0}\left(s_{1}\right), h_{1}\left(s_{1}\right)$ - положительные производящие функции. На рис. 1 , случай в) (см. с. 453) изображены возможные скачки случайного блуждания $S_{n}$. 
Лемма 4. Пусть $h\left(s_{1}, s_{2}\right)=h_{0}\left(s_{1}\right)+s_{2} h_{1}\left(s_{1}\right)$, причем для распределения $\left\{p_{\gamma_{1} \gamma_{2}},\left(\gamma_{1}, \gamma_{2}\right) \in \mathbf{N}^{2}\right\}$ имеются вероятности $p_{0 \gamma_{2}}>0, p_{\gamma_{1} \gamma_{2}}>0$ с $\gamma_{1} \geqslant 2$ и $p_{\gamma_{1} 0}>0$. Тогда функиия $\varphi_{1}(s)$ является аналитической в области $|s|>r_{2}, 0<r_{2} \leqslant 1$, и значение $\varphi_{1}\left(r_{2}\right)$ лежит в интервале $\left(r_{1}, R_{1}\right), 0 \leqslant r_{1}<1<R_{1} \leqslant \infty$.

При условиях леммы 4 решетка $S_{L}$ двумерна. Доказательство леммы 4 аналогично доказательству лемм 2 и 3 , при этом используется явное выражение $\varphi_{2}(s)=h_{0}(s) /\left(s-h_{1}(s)\right)$, которое следует из уравнения $h_{0}\left(s_{1}\right)+s_{2} h_{1}\left(s_{1}\right)-s_{1} s_{2}=0$.

Теорема 3. Пусть $h\left(s_{1}, s_{2}\right)=h_{0}\left(s_{1}\right)+s_{2} h_{1}\left(s_{1}\right)$ и вылполнены условия леммы 4. Вероятность остановки равна $\left(\alpha_{1} \in \mathbf{N}, \alpha_{2}=1,2, \ldots\right)$

$$
q_{\left(0, \gamma_{2}\right)}^{\left(\alpha_{1}, \alpha_{2}\right)}=\frac{1}{2 \pi i} \int_{\theta_{\rho}^{+}} \varphi_{1}^{\alpha_{1}}(s) s^{\alpha_{2}} \frac{d s}{s^{\gamma_{2}+1}}, \quad \gamma_{2}=1,2, \ldots,
$$

где контур интегрирования - окружность $\theta_{\rho}:|s|=\rho, \rho>r_{2}$.

Д о к а з а т е л с т в о. Исходим из интегрального представления $(20)$. Вместо $\Phi_{2}^{\left(\alpha_{1}, \alpha_{2}\right)}(s)$ подставляем $(17)$, получаем $\left(\gamma_{2}=1,2, \ldots\right)$

$$
q_{\left(0, \gamma_{2}\right)}^{\left(\alpha_{1}, \alpha_{2}\right)}=\frac{1}{2 \pi i} \int_{\theta_{\rho}^{+}} \varphi_{1}^{\alpha_{1}}(s) s^{\alpha_{2}} \frac{d s}{s^{\gamma_{2}+1}}-\frac{1}{2 \pi i} \int_{\theta_{\rho}^{+}} \Phi_{1}^{\left(\alpha_{1}, \alpha_{2}\right)}\left(\varphi_{1}(s)\right) \frac{d s}{s^{\gamma_{2}+1}},
$$

где $\rho>r_{2}$. Второй интеграл равен нулю, так как из условия $h\left(s_{1}, s_{2}\right)=$ $h_{0}\left(s_{1}\right)+s_{2} h_{1}\left(s_{1}\right)$ на скачки случайного блуждания $S_{n}$ следует, что разложение функции $\varphi_{1}(s)$ в ряд Лорана в окрестности $|s|>r_{2}$ бесконечно удаленной точки не содержит главной части,

$$
\varphi_{1}(s)=\widehat{q}_{(0,0)}^{(1,0)}+\frac{\widehat{q}_{(0,-1)}^{(1,0)}}{s}+\frac{\widehat{q}_{(0,-2)}^{(1,0)}}{s^{2}}+\cdots ;
$$

а следовательно, (как показывается, исходя из неравенств $\varphi_{1}\left(r_{2}\right)<R_{1}$ и $\varphi_{1}(s)<R_{1}$ при $\left.|s|>r_{2}\right)$ и разложение функции $\Phi_{1}^{\left(\alpha_{1}, \alpha_{2}\right)}\left(\varphi_{1}(s)\right)$ в ряд Лорана в области $|s|>r_{2}$ не содержит главной части. Теорема 3 доказана.

7. Интегральное представление для вероятностей $q_{\left(0, \gamma_{2}\right)}^{\left(\alpha_{1}, \alpha_{2}\right)}$ в случае $h\left(s_{1}, s_{2}\right)=h_{0}\left(s_{2}\right)+s_{1} h_{1}\left(s_{2}\right)$. Положим $h\left(s_{1}, s_{2}\right)=h_{0}\left(s_{2}\right)+s_{1} h_{1}\left(s_{2}\right)$, где $h_{0}\left(s_{2}\right), h_{1}\left(s_{2}\right)$ - положительные производящие функции. На рис. 3 , случай а), изображены возможные скачки случайного блуждания $S_{n}$.

Лемма 5. Пусть $h\left(s_{1}, s_{2}\right)=h_{0}\left(s_{2}\right)+s_{1} h_{1}\left(s_{2}\right)$, причем для распределения $\left\{p_{\gamma_{1} \gamma_{2}},\left(\gamma_{1}, \gamma_{2}\right) \in \mathbf{N}^{2}\right\}$ имеются вероятности $p_{0 \gamma_{2}}>0, p_{\gamma_{1} \gamma_{2}}>0$ с $\gamma_{2} \geqslant 2$ и $p_{\gamma_{1} 0}>0$. Тогда: Функиия $\varphi_{1}(s)$ является аналитической в кольче $r_{2}<|s|<R_{2}, 0 \leqslant r_{2}<1<R_{2} \leqslant \infty, u \lim _{s \downarrow r_{2}} \varphi_{1}(s)=+\infty$, $\lim _{s \uparrow R_{2}} \varphi_{1}(s)=+\infty ;$ бункиия $\varphi_{2}(s)$ является аналитической в области $|s|>r_{1}, 0<r_{1} \leqslant 1$, и значение $\tilde{r}_{2}=\varphi_{2}\left(r_{1}\right)$ лежит в интервале $\left(r_{2}, R_{2}\right)$. 


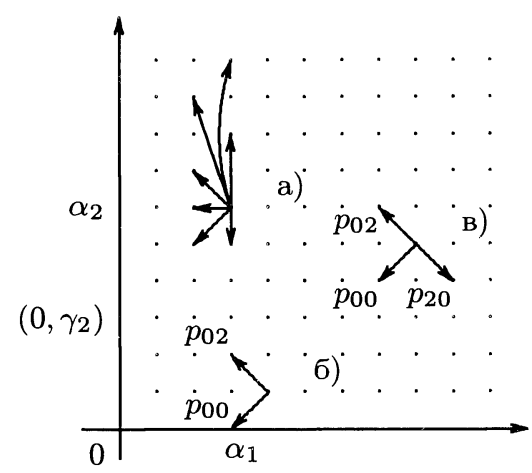

Рис. 3

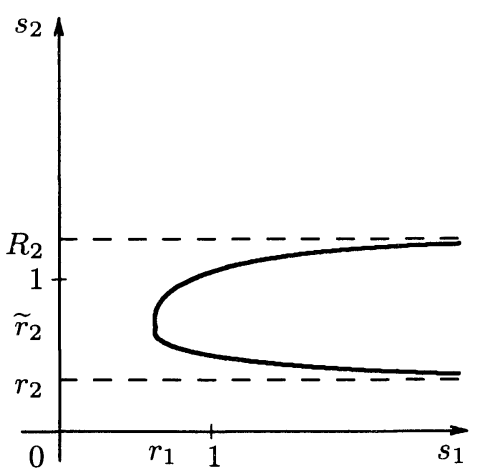

Рис. 4

При условиях леммы 5 решетка $S_{L}$ двумерна. Доказательство леммы 5 аналогично доказательству лемм 2 и 3 , при этом используется выражение $\varphi_{1}(s)=h_{0}(s) /\left(s-h_{1}(s)\right)$, которое следует из характеристического уравнения. Множество $Q^{+}$изображено на рис. 4.

Ниже мы будем использовать следующие обозначения. Характеристическое уравнение $h\left(s_{1}, s_{2}\right)-s_{1} s_{2}=0$ определяет некоторую многозначную функцию комплексного аргумента. Рассмотренную в лемме 5 однозначную ветвь этой функции в области $|s|>r_{1}$ обозначим $\widetilde{\varphi}_{2}(s)$ (таким образом, $\tilde{\varphi}_{2}(s)=\varphi_{2}^{(0,1)}(s)$, см. (11)). Так же, как в [2, гл. 5, $\S 4]$, показывается, что точка $s=r_{1}$ является изолированной точкой ветвления второго порядка; можно показать, учитывая лемму 5, что для области $|s|>r_{1}$ имеется вторая однозначная ветвь $\bar{\varphi}_{2}(s)$, выделяемая условием $\lim _{s \downarrow r_{1}} \bar{\varphi}_{2}(s)=\widetilde{r}_{2}$. Обозначим $\varphi_{2}(s)$ двузначную ветвь в области $\left\{|s|>r_{1}\right\} \cup\left\{0<\left|s-r_{1}\right|<\varepsilon\right\}$, составленную из ветвей $\widetilde{\varphi}_{2}(s), \bar{\varphi}_{2}(s)$ и аналитического продолжения этих ветвей в кольцо $0<\left|s-r_{1}\right|<\varepsilon(\varepsilon>0$ достаточно мало, поэтому круг $\left|s-r_{1}\right|<\varepsilon$ не содержит особых точек, кроме $r_{1}$ ).

Определим при $r_{2}<|s|<\widetilde{r}_{2}$ функцию $\bar{\psi}_{21}(s)=\bar{\varphi}_{2}\left(\varphi_{1}(s)\right)$ и при $\tilde{r}_{2}<|s|<R_{2}$ функцию $\widetilde{\psi}_{21}(s)=\tilde{\varphi}_{2}\left(\varphi_{1}(s)\right.$ ) (корректность определения следует из неравенства $\left|\varphi_{1}(s)\right|>r_{1}$ при $\left.r_{2}<|s|<R_{2}\right)$, для которых $h\left(\varphi_{1}(s), \bar{\psi}_{21}(s)\right)-\varphi_{1}(s) \bar{\psi}_{21}(s)=0$ и $h\left(\varphi_{1}(s), \widetilde{\psi}_{21}(s)\right)-\varphi_{1}(s) \widetilde{\psi}_{21}(s)=0$. Уравнение $h\left(\varphi_{1}(s), s_{2}\right)-\varphi_{1}(s) s_{2}=0$ после подстановки выражений $h\left(s_{1}, s_{2}\right)=h_{0}\left(s_{2}\right)+s_{1} h_{1}\left(s_{2}\right)$ и $\varphi_{1}(s)=h_{0}(s) /\left(s-h_{1}(s)\right)$ получает простой вид:

$$
\frac{h_{0}(s)}{s-h_{1}(s)}=\frac{h_{0}\left(s_{2}\right)}{s_{2}-h_{1}\left(s_{2}\right)} .
$$

Используя применительно к последнему уравнению теорему о неявной функции для нахождения особых точек, устанавливаем, что аналитическим продолжением функции $\bar{\psi}_{21}(s)$ в кольцо $r_{2}<|s|<R_{2}$ является, для области $\widetilde{r}_{2}<|s|<R_{2}$, функция $\widetilde{\psi}_{21}(s)$; и более того, существует ана- 
литическое продолжение на область $|s|>r_{2}$, которое обозначим $\psi_{21}(s)$ (для $\psi_{21}(s)$ справедливо соотношение $h\left(\varphi_{1}(s), \psi_{21}(s)\right)-\varphi_{1}(s) \psi_{21}(s)=0$ ). Очевидно, $\widetilde{\psi}_{21}\left(\bar{\psi}_{21}(s)\right)=s$ при $r_{2}<|s|<\widetilde{r}_{2}$ и $\bar{\psi}_{21}\left(\widetilde{\psi}_{21}(s)\right)=s$ при $\tilde{r}_{2}<|s|<R_{2}$; следовательно, по принципу консерватизма функциональных уравнений $\psi_{21}\left(\psi_{21}(s)\right)=s$ при $|s|>r_{2}$, т.е. функция $\psi_{21}(s)$ однолистна на множестве $|s|>r_{2}$ (по признаку однолистности функции, данному в $[10$, гл. $5, \S 1])$. Отсюда следует, что разложение функции $\psi_{21}(s)$ в ряд Лорана в области $|s|>r_{2}$ не содержит главной части ([10, гл. $5, \S 1$, следствие 1$]$; ср. п. 3 доказательства теоремы 5.1 гл. 5 в $[10])$.

Ниже для производной функции двух переменных $H\left(s_{1}, s_{2}\right)$ используется обозначение $\left(H\left(s_{1}, s_{2}\right)\right)_{s_{1}}^{\prime}=\partial H\left(s_{1}, s_{2}\right) / \partial s_{1}$.

Теорема 4. Пусть $h\left(s_{1}, s_{2}\right)=h_{0}\left(s_{2}\right)+s_{1} h_{1}\left(s_{2}\right)$ и вылолненьл условия леммь 5. Вероятность остановки равна $\left(\alpha_{1} \in \mathbf{N}, \alpha_{2}=1,2, \ldots\right)$

$$
q_{\left(0, \gamma_{2}\right)}^{\left(\alpha_{1}, \alpha_{2}\right)}=\frac{1}{2 \pi i} \int_{\theta_{\rho}^{+}}\left(\frac{1}{2 \pi i} \int_{\Theta} s_{1}^{\alpha_{1}} \varphi_{2}^{\alpha_{2}}\left(s_{1}\right) \frac{\left(h\left(s_{1}, s_{2}\right)-s_{1} s_{2}\right)_{s_{1}}^{\prime}}{h\left(s_{1}, s_{2}\right)-s_{1} s_{2}} d s_{1}\right) \frac{d s_{2}}{s_{2}^{\gamma_{2}+1}}
$$

$\gamma_{2}=1,2, \ldots$, где $r_{2}<\rho<\widetilde{r}_{2} ;$ контур интегрирования $\Theta$ описан ниже.

Д о к а з а т е л ь с т в о. Ветвь $\bar{\varphi}_{2}(s)$ получена как аналитическое продолжение ветви $\tilde{\varphi}_{2}(s)$ при обходе точки ветвления $r_{1}$ вдоль кривой $\theta(t)=r_{1}+\varepsilon_{1} e^{i t}, 0 \leqslant t \leqslant 2 \pi$, где $0<\varepsilon_{1}<\varepsilon$. При достаточно малом $\varepsilon_{1}$ значения функции $\varphi_{2}(s)$ на кривой $\theta(t)$ лежат в области аналитичности $|s|<R_{2}$ функции $\Phi_{2}^{\left(\alpha_{1}, \alpha_{2}\right)}(s)$; из условия $h\left(s_{1}, s_{2}\right)=h_{0}\left(s_{2}\right)+s_{1} h_{1}\left(s_{2}\right)$ на скачки случайного блуждания $S_{n}$ следует, что $\Phi_{1}^{\left(\alpha_{1}, \alpha_{2}\right)}(s)-$ многочлен, т.е. $\Phi_{1}^{\left(\alpha_{1}, \alpha_{2}\right)}(s)$ аналитична на всей комплексной плоскости, - следовательно, по принципу консерватизма функциональных уравнений, $\bar{\varphi}_{2}(s)$ удовлетворяет функциональному уравнению (16):

$$
\Phi_{1}^{\left(\alpha_{1}, \alpha_{2}\right)}(s)=s^{\alpha_{1}} \bar{\varphi}_{2}^{\alpha_{2}}(s)-\Phi_{2}^{\left(\alpha_{1}, \alpha_{2}\right)}\left(\bar{\varphi}_{2}(s)\right)+\Phi_{2}^{\left(\alpha_{1}, \alpha_{2}\right)}(0), \quad|s|>r_{1} .
$$
$\left.\Phi_{2}^{\left(\alpha_{1}, \alpha_{2}\right)}(0)\right)$

$$
\Phi_{2}^{\left(\alpha_{1}, \alpha_{2}\right)}(s)=\varphi_{1}^{\alpha_{1}}(s) s^{\alpha_{2}}-\varphi_{1}^{\alpha_{1}}(s) \bar{\varphi}_{2}^{\alpha_{2}}\left(\varphi_{1}(s)\right)+\Phi_{2}^{\left(\alpha_{1}, \alpha_{2}\right)}\left(\bar{\varphi}_{2}\left(\varphi_{1}(s)\right)\right)
$$

при $r_{2}<|s|<\tilde{r}_{2}$. Интегральное представление $(20)$ для искомых вероятностей после подстановки (23) получает вид

$$
\begin{aligned}
q_{\left(0, \gamma_{2}\right)}^{\left(\alpha_{1}, \alpha_{2}\right)}= & \frac{1}{2 \pi i} \int_{\theta_{\rho}^{+}}\left(\varphi_{1}^{\alpha_{1}}(s) s^{\alpha_{2}}-\varphi_{1}^{\alpha_{1}}(s) \bar{\varphi}_{2}^{\alpha_{2}}\left(\varphi_{1}(s)\right)\right) \frac{d s}{s^{\gamma_{2}+1}} \\
& +\frac{1}{2 \pi i} \int_{\theta_{\rho}^{+}} \Phi_{2}^{\left(\alpha_{1}, \alpha_{2}\right)}\left(\bar{\varphi}_{2}\left(\varphi_{1}(s)\right)\right) \frac{d s}{s^{\gamma_{2}+1}}, \quad \gamma_{2}=1,2, \ldots
\end{aligned}
$$


где $r_{2}<\rho<\widetilde{r}_{2}$. Второй интеграл равен нулю - выше отмечалось, что функция $\bar{\varphi}_{2}\left(\varphi_{1}(s)\right)$ может быть аналитически продолжена в область $|s|>r_{2}$ и ее разложение в ряд Лорана в окрестности бесконечно удаленной точки не содержит главной части, a, следовательно, и разложение функции $\Phi_{2}^{\left(\alpha_{1}, \alpha_{2}\right)}\left(\bar{\varphi}_{2}\left(\varphi_{1}(s)\right)\right)$ в ряд Лорана в области $|s|>r_{2}$ не содержит главной части.

Используя интегральную формулу Коши, получаем равенство (здесь $\tilde{\theta}_{\rho}$ - окружность $\left|s_{1}-\varphi_{1}(s)\right|=\rho$, где радиус $\rho>0$ достаточно мал, чтобы $\tilde{\theta}_{\rho}$ лежала в области $\left.\left|s_{1}\right|>r_{1}\right)$

$$
\begin{aligned}
& \varphi_{1}^{\alpha_{1}}(s) s^{\alpha_{2}}-\varphi_{1}^{\alpha_{1}}(s) \bar{\varphi}_{2}^{\alpha_{2}}\left(\varphi_{1}(s)\right)=\frac{1}{2 \pi i} \int_{\tilde{\theta}_{\rho}^{+}} s_{1}^{\alpha_{1}} \widetilde{\varphi}_{2}^{\alpha_{2}}\left(s_{1}\right) \frac{d s_{1}}{s_{1}-\varphi_{1}(s)} \\
& -\frac{1}{2 \pi i} \int_{\tilde{\theta}_{\rho}^{+}} s_{1}^{\alpha_{1}} \bar{\varphi}_{2}^{\alpha_{2}}\left(s_{1}\right) \frac{d s_{1}}{s_{1}-\varphi_{1}(s)}=\frac{1}{2 \pi i} \int_{\Theta} s_{1}^{\alpha_{1}} \varphi_{2}^{\alpha_{2}}\left(s_{1}\right) \frac{d s_{1}}{s_{1}-\varphi_{1}(s)}
\end{aligned}
$$

(учитывая результаты леммы 5 , нетрудно показать, что $\widetilde{\varphi}_{2}\left(\varphi_{1}(s)\right) \equiv s$ при $\left.r_{2}<|s|<\tilde{r}_{2}\right)$, где $\Theta$ обозначает следующий контур Похгаммера. Замкнутый контур начинается в точке вещественной оси $r_{0}=r_{1}+\rho_{1}$, $0<\rho_{1}<\varepsilon$, и состоит из лежащей в области $\left|s_{1}\right|>r_{1}$ простой кривой $\theta^{+}$, соединяющей точку $r_{0}$ и некоторую точку окружности $\tilde{\theta}_{\rho}$; окружности $\tilde{\theta}_{\rho}^{+} ;$кривой $\theta^{-}$; окружности $\hat{\theta}_{\rho_{1}}^{+}:\left|s_{1}-r_{1}\right|=\rho_{1}$ радиуса $\rho_{1} ;$ кривой $\theta^{+}$; окружности $\tilde{\theta}_{\rho}^{-}$; кривой $\theta^{-}$; окружности $\hat{\theta}_{\rho_{1}}^{-}$. При обходе контура $\Theta$ функция $\varphi_{2}\left(s_{1}\right)$ получает в точке $r_{0}$ свое первоначальное значение. Подставляя выражение (25) в (24), получаем (переменную $s$ в формуле (25) обозначим $s_{2}$ )

$$
q_{\left(0, \gamma_{2}\right)}^{\left(\alpha_{1}, \alpha_{2}\right)}=\frac{1}{2 \pi i} \int_{\theta_{\rho}^{+}}\left(\frac{1}{2 \pi i} \int_{\Theta} s_{1}^{\alpha_{1}} \varphi_{2}^{\alpha_{2}}\left(s_{1}\right) \frac{d s_{1}}{s_{1}-\varphi_{1}\left(s_{2}\right)}\right) \frac{d s_{2}}{s_{2}^{\gamma_{2}+1}},
$$

где $r_{2}<\rho<\widetilde{r}_{2}$. Подставляя функцию

$$
\varphi_{1}\left(s_{2}\right)=\frac{h_{0}\left(s_{2}\right)}{s_{2}-h_{1}\left(s_{2}\right)}
$$

в дробь в подынтегральном выражении:

$$
\frac{1}{s_{1}-\varphi_{1}\left(s_{2}\right)}=\frac{s_{2}-h_{1}\left(s_{2}\right)}{s_{1} s_{2}-s_{1} h_{1}\left(s_{1}\right)-h_{0}\left(s_{2}\right)}=\frac{\left(h\left(s_{1}, s_{2}\right)-s_{1} s_{2}\right)_{s_{1}}^{\prime}}{h\left(s_{1}, s_{2}\right)-s_{1} s_{2}}
$$

приходим к формуле (22). Теорема 4 доказана.

П р и м е р 1. Пусть $h\left(s_{1}, s_{2}\right)=p_{02} s_{2}^{2}+p_{00}$ (рис. 3 , случай б) с. 464$)$. Тогда вероятность $q_{\left(0, \gamma_{2}\right)}^{\left(\alpha_{1}, \alpha_{2}\right)}$ можно интерпретировать как вероятность попадания - для случайного блуждания на множестве $\mathbf{N}=\{0,1, \ldots\}$ с вероятностью $p_{00}$ скачка влево, с вероятностью $p_{02}$ скачка вправо и поглощением на границе 0 - из начального состояния $\alpha_{2}$ в состояние $\gamma_{2}$ 
на $\alpha_{1}$-шаге блуждания. Такое случайное блуждание рассмотрено в [7, гл. 14 , задача 20]. Формулы $(4.8)$ ([7, гл. 14]) указывают на переход к рассмотрению ветвей многозначной функции, определяемой уравнением $h\left(s_{1}, s_{2}\right)-s_{1} s_{2}=0$. Приведение результатов [7] для $q_{\left(0, \gamma_{2}\right)}^{\left(\alpha_{1}, \alpha_{2}\right)}$ к виду $(22)$ дает следующее представление:

$$
q_{\left(0, \gamma_{2}\right)}^{\left(\alpha_{1}, \alpha_{2}\right)}=\frac{1}{2 \pi i} \int_{\theta_{\rho}^{-}} s^{\alpha_{1}} \tilde{\varphi}_{2}^{\alpha_{2}}(s)\left(-\frac{2}{\gamma_{2}}\right) d T_{\gamma_{2}}(s),
$$

где $\rho>r_{1}, \widetilde{\varphi}_{2}(s)=\left(s-\sqrt{s^{2}-4 p_{00} p_{02}}\right) /\left(2 p_{02}\right)\left(r_{1}=2 \sqrt{p_{00} p_{02}}\right)$,

$$
T_{1}(s)=\frac{s}{2 p_{00}}, \quad T_{2}(s)=\frac{s^{2}-2 p_{00} p_{02}}{2 p_{00}^{2}}, \quad T_{3}(s)=\frac{s^{3}-3 p_{00} p_{02} s}{2 p_{00}^{3}}, \ldots
$$

- многочлены Чебышева, определяемые на отрезке $\left[-2 \sqrt{p_{00} p_{02}}, 2 \sqrt{p_{00} p_{02}}\right]$ разложением $\left(-2 \sqrt{p_{00} p_{02}}<s_{1}<2 \sqrt{p_{00} p_{02}},\left|s_{2}\right|<\sqrt{p_{00} / p_{02}}\right)$

$\ln \left(h\left(s_{1}, s_{2}\right)-s_{1} s_{2}\right)=\ln \left(p_{02} s_{2}^{2}+p_{00}-s_{1} s_{2}\right)=\ln p_{00}+\sum_{\gamma_{2}=1}^{\infty}\left(-\frac{2}{\gamma_{2}}\right) T_{\gamma_{2}}\left(s_{1}\right) s_{2}^{\gamma_{2}}$

(см. $[16$, гл. $10, \S 11,(30)])$. Вычислим по формуле $(26)$ вероятность $q_{(0,3)}^{(4,1)}$, используя разложение функции $\widetilde{\varphi}_{2}(s)$ в ряд Лорана в окрестности $|s|>r_{1}$ точки $s=\infty$ (см. [9, гл. IV, $\S 24$, пример 11]):

$$
\begin{aligned}
q_{(0,3)}^{(4,1)}= & \frac{1}{2 \pi i} \int_{\theta_{\rho}^{-}} s^{4} \widetilde{\varphi}_{2}(s)\left(-\frac{2}{3}\right) d T_{3}(s) \\
= & \frac{1}{2 \pi i} \int_{\theta_{\rho}^{-}} s^{4} \cdot s \frac{1-\sqrt{1-4 p_{00}} p_{02} / s^{2}}{2 p_{02}}\left(-\frac{2}{3}\right) d\left(\frac{s^{3}-3 p_{00} p_{02} s}{2 p_{00}^{3}}\right) \\
= & \frac{1}{2 \pi i} \int_{\theta_{\rho}^{+}} s^{4}\left(\frac{p_{00}}{s}+\frac{p_{00}^{2} p_{02}}{s^{3}}+\frac{2 p_{00}^{3} p_{02}^{2}}{s^{5}}+\frac{5 p_{00}^{4} p_{02}^{3}}{s^{7}}+\cdots\right) \\
& \quad \times\left(\frac{s^{2}-p_{00} p_{02}}{p_{00}^{3}}\right) d s=\frac{5 p_{00}^{4} p_{02}^{3}-2 p_{00}^{4} p_{02}^{3}}{p_{00}^{3}}=3 p_{00} p_{02}^{3} .
\end{aligned}
$$

То же значение $q_{(0,3)}^{(4,1)}$ можно получить из рассмотрения рис. 3 (с. 464$)$.

8. Интегральное представление для вероятностей $q_{\left(0, \gamma_{2}\right)}^{\left(\alpha_{1}, \alpha_{2}\right)}$ в случае $h\left(s_{1}, s_{2}\right)=p_{20} s_{1}^{2}+p_{02} s_{2}^{2}+p_{00}$. Пусть $\omega$ и $\omega^{\prime}-$ положительные числа. Положим $h=\exp \left\{-\pi \omega^{\prime} / \omega\right\}, z=\exp \{\pi i u /(2 \omega)\}$. Определим двоякопериодическую функцию равенством $(\gamma=1,2, \ldots)$

$$
f_{\gamma}(u)=\left(\frac{\pi i}{\omega}\right)^{\gamma}\left\{\frac{1}{\left(z-z^{-1}\right)^{\gamma}}+\sum_{r=1}^{\infty}\left(\frac{h^{r} z^{-1}}{1-h^{2 r} z^{-2}}\right)^{\gamma}+\sum_{r=1}^{\infty}\left(\frac{-h^{r} z}{1-h^{2 r} z^{2}}\right)^{\gamma}\right\} .
$$


При нечетных $\gamma$ периодами являются $4 \omega, 2 i \omega^{\prime}$, при четных $\gamma$ периодами являются $2 \omega, 2 i \omega^{\prime}$. Сходимость ряда вида $(27)$ рассмотрена в $[13$, гл. 2 , $\S 12]$. Из (27) легко получить разложение в ряд Фурье

$$
\begin{aligned}
f_{\gamma}(u)=\left(\frac{\pi}{\omega}\right)^{\gamma} & \left\{\frac{1}{2^{\gamma}} \operatorname{cosec}^{\gamma}\left(\frac{\pi u}{2 \omega}\right)+(-1)^{[\gamma / 2]} 2\right. \\
& \left.\times \sum_{n=1}^{\infty} C_{n+\gamma-1}^{\gamma-1} \frac{h^{2 n+\gamma}}{1-h^{2 n+\gamma}}\left\{\begin{array}{c}
\cos \\
\sin
\end{array}\right\}\left(\frac{\pi u}{2 \omega}(2 n+\gamma)\right)\right\},
\end{aligned}
$$

где знак $\cos$ берется при четных $\gamma$, знак $\sin$ берется при нечетных $\gamma$. Разложение (28) годно в полосе $\operatorname{Im} u<2 \omega^{\prime}$, за исключением полюсов первого слагаемого (см. $[12, \S 22.61$, задача 20.35]). Сравнивая (28) с известными разложениями в ряды Фурье эллиптических функций Якоби (см. [12, § 22.61, задача 22.57]), получаем

$$
f_{\gamma}(u)=\frac{c_{\gamma}}{(\operatorname{sn} c u)^{\gamma}}+\frac{c_{\gamma-2}}{(\operatorname{sn} c u)^{\gamma-2}}+\cdots+c_{0},
$$

где $c, c_{\gamma}, \ldots$ - некоторые константы. Функция $f_{2}(u)$ с точностью до постоянного слагаемого совпадает с эллиптической функцией Вейерштрасса [13].

Теорема 5. Пусть $h\left(s_{1}, s_{2}\right)=p_{20} s_{1}^{2}+p_{02} s_{2}^{2}+p_{00}$. Положим

$$
C=\frac{1-\sqrt{1-4 p_{20} p_{02}}}{1+\sqrt{1-4 p_{20} p_{02}}}, \quad C_{0}=\frac{p_{20} p_{00}}{1-4 p_{20} p_{02}} ;
$$

$f_{\gamma_{2}}(u)-$ эллиптическая функиия $(27) c \omega=\pi \sqrt{C_{0}}, \omega^{\prime}=-\sqrt{C_{0}} \ln C ; T=$ $\left\{u=x+i y, 0 \leqslant x \leqslant 4 \omega, y=-\omega^{\prime}\right\}-$ ориентированный по возрастанию $x$ отрезок. Вероятность остановки равна $\left(\left(\alpha_{1}, \alpha_{2}\right) \in \mathbf{N}^{2}\right)$

$$
q_{\left(0, \gamma_{2}\right)}^{\left(\alpha_{1}, \alpha_{2}\right)}=\frac{1}{2 \pi i} \int_{T} s_{1}^{\alpha_{1}}\left(e^{\pi i u /(2 \omega)}\right) s_{2}^{\alpha_{2}}\left(e^{\pi i u /(2 \omega)}\right) \frac{(-1)^{\gamma_{2}}}{-\gamma_{2}} d f_{\gamma_{2}}(u),
$$

$\gamma_{2}=1,2, \ldots$, где функиии

$$
s_{1}(z)=\frac{1-\sqrt{1-4 p_{20} p_{02}}}{2 p_{20}} i \sqrt{C_{0}}\left(z-\frac{1}{C z}\right), \quad s_{2}(z)=i \sqrt{C_{0}}\left(z-\frac{1}{z}\right)
$$

представляют собой униформизацию римановой поверхности (6).

На рис. 3 , случай в), изображены возможные скачки случайного блуждания $S_{n}$. Пример вычисления вероятности остановки по формуле (29) дан в $[20]$.

Д о к а з а т е л ь с т в о. Уравнение (4) для экспоненциальной производящей функции вероятностей $q_{\left(0, \gamma_{2}\right)}^{\left(\alpha_{1}, \alpha_{2}\right)}$ получает вид:

$$
\left(p_{20} \frac{\partial^{2}}{\partial z_{1}^{2}}+p_{02} \frac{\partial^{2}}{\partial z_{2}^{2}}+p_{00}-\frac{\partial^{2}}{\partial z_{1} \partial z_{2}}\right) g_{\left(0, \gamma_{2}\right)}\left(z_{1}, z_{2}\right)=0
$$


с граничными условиями (5); соответствующее характеристическое уравнение имеет вид

$$
p_{20} s_{1}^{2}+p_{02} s_{2}^{2}+p_{00}-s_{1} s_{2}=0
$$

А). Построение незамкнутого решения задачи Дарбу-Пикаpa (31), (5). Уравнение второго порядка (31) принадлежит гиперболическому типу ([14], [15]). Находим общие интегралы уравнений характеристик; вводя вместо $z_{1}, z_{2}$ новые независимые переменные $x, y$ :

$$
\begin{aligned}
& x=\frac{1-\sqrt{1-4 p_{20} p_{02}}}{2 p_{20}} z_{1}+z_{2}, \\
& y=\frac{p_{00} p_{20}}{1-4 p_{20} p_{02}}\left(\frac{1+\sqrt{1-4 p_{20} p_{02}}}{2 p_{20}} z_{1}+z_{2}\right),
\end{aligned}
$$

приводим уравнение (31) к каноническому виду:

$$
\frac{\partial^{2} g_{\left(0, \gamma_{2}\right)}}{\partial x \partial y}=g_{\left(0, \gamma_{2}\right)}
$$

(так называемое «телеграфное уравнение»). При преобразовании (33) прямые $z_{1}=0$ и $z_{2}=0$ переходят, соответственно, в прямые $y=C_{0} x$, $y=\left(C_{0} / C\right) x$; граничным условиям (5) соответствуют условия для (34):

$$
\left.g_{\left(0, \gamma_{2}\right)}(x, y)\right|_{y=C_{0} x}=\frac{x^{\gamma_{2}}}{\gamma_{2} !},\left.\quad g_{\left(0, \gamma_{2}\right)}(x, y)\right|_{y=C_{0} x / C}=0
$$

Таким образом, для уравнения (34) решаем задачу Дарбу-Пикара: заданы условия (35) на двух монотонных кривых, выходящих из точки $(0,0)$ и расположенных в характеристическом угле $x=0, y=0$ с вершиной в той же точке $(0,0)$. Из теории уравнений в частных производных второго порядка гиперболического типа известно, что решение рассматриваемой задачи (34), (35) существует и единственно (ср. условие (3) аналитичности искомого решения). Для построения решения задачи (34), (35) воспользуемся общим решением телеграфного уравнения [14, задача 30]:

$$
\begin{aligned}
g_{\left(0, \gamma_{2}\right)}(x, y)= & \int_{0}^{x} \psi_{1}(t) J_{0}(2 i \sqrt{y(x-t)}) d t \\
& +\int_{0}^{y} \psi_{2}(t) J_{0}(2 i \sqrt{x(y-t)}) d t+g_{\left(0, \gamma_{2}\right)}(0,0) J_{0}(2 i \sqrt{x y}),(36
\end{aligned}
$$

где $\psi_{1}(t)$ и $\psi_{2}(t)$ - произвольные функции $\left(J_{0}(t)\right.$ - функция Бесселя нулевого порядка). Выражение (36) представляет собой формулу Римана [15] для решения уравнения (34). 
При условии $\gamma_{2}=1,2, \ldots$ имеем $g_{\left(0, \gamma_{2}\right)}(0,0)=0$. Найдем функции $\psi_{1}(t)$ и $\psi_{2}(t)$ такие, чтобы выполнялись условия (35). При подстановке в (36) второго из условий (35) получаем

$$
\begin{aligned}
& \left.g_{\left(0, \gamma_{2}\right)}(x, y)\right|_{y=C_{0} x / C}=\int_{0}^{x} \psi_{1}(t) J_{0}\left(2 i \sqrt{\frac{C_{0}}{C} x(x-t)}\right) d t \\
& \quad+\int_{0}^{C_{0} x / C} \psi_{2}(t) J_{0}\left(2 i \sqrt{x\left(\frac{C_{0}}{C} x-t\right)}\right) d t \\
& =\int_{0}^{x}\left(\psi_{1}(t)+\frac{C_{0}}{C} \psi_{2}\left(\frac{C_{0}}{C} t\right)\right) J_{0}\left(2 i \sqrt{\frac{C_{0}}{C} x(x-t)}\right) d t=0 .
\end{aligned}
$$

Отсюда следует, что $\psi_{2}(t)=-\left(C / C_{0}\right) \psi_{1}\left(\left(C / C_{0}\right) t\right)$. Заменяя в $(36) \psi_{2}(t)$ последним выражением и затем подставляя в (36) первое из условий $(35)$, получаем

$$
\begin{aligned}
\left.g_{\left(0, \gamma_{2}\right)}(x, y)\right|_{y=C_{0} x}= & \int_{0}^{x} \psi_{1}(t) J_{0}\left(2 i \sqrt{C_{0} x(x-t)}\right) d t \\
& -\int_{0}^{C_{0} x} \frac{C}{C_{0}} \psi_{1}\left(\frac{C}{C_{0}} t\right) J_{0}\left(2 i \sqrt{x\left(C_{0} x-t\right)}\right) d t=\frac{x^{\gamma_{2}}}{\gamma_{2} !} .
\end{aligned}
$$

Или, после замены переменной во втором слагаемом,

$$
\frac{x^{\gamma_{2}}}{\gamma_{2} !}=\int_{0}^{x}\left(\psi_{1}(t)-C \psi_{1}(C t)\right) J_{0}\left(2 i \sqrt{C_{0} x(x-t)}\right) d t .
$$

Функцию $\psi_{1}(t)$ ищем в виде ряда $\psi_{1}(t)=\sum_{k=0}^{\infty} a_{\gamma_{2}-1+2 k} t^{\gamma_{2}-1+2 k}$. Тогда (37) получает вид:

$$
\frac{x^{\gamma_{2}}}{\gamma_{2} !}=\int_{0}^{x}\left(\sum_{k=0}^{\infty} a_{\gamma_{2}-1+2 k}\left(1-C^{\gamma_{2}+2 k}\right) t^{\gamma_{2}-1+2 k}\right) J_{0}\left(2 i \sqrt{C_{0} x(x-t)}\right) d t .
$$

Учитывая значения интегралов (ср. [17, интеграл 6.567.1])

$$
\int_{0}^{x} t^{\gamma_{2}-1+2 k} J_{0}\left(2 i \sqrt{C_{0} x(x-t)}\right) d t=\frac{\left(\gamma_{2}-1+2 k\right) !}{\left(i \sqrt{C_{0}}\right)^{\gamma_{2}+2 k}} J_{\gamma_{2}+2 k}\left(2 i \sqrt{C_{0}} x\right),
$$

где $k=0,1, \ldots$, получаем

$$
\frac{x^{\gamma_{2}}}{\gamma_{2} !}=\sum_{k=0}^{\infty} a_{\gamma_{2}-1+2 k} \frac{\left(1-C^{\gamma_{2}+2 k}\right)\left(\gamma_{2}-1+2 k\right) !}{\left(i \sqrt{C_{0}}\right)^{\gamma_{2}+2 k}} J_{\gamma_{2}+2 k}\left(2 i \sqrt{C_{0}} x\right) .
$$

Сравнивая последнее выражение с рядом Неймана $[16, \S 7.10 .1]$

$$
x^{\gamma_{2}}=\sum_{k=0}^{\infty} \frac{\left(\gamma_{2}+2 k\right)\left(\gamma_{2}+k-1\right) !}{k !} J_{\gamma_{2}+2 k}(2 x),
$$


находим выражения для коэффициентов $a_{\gamma_{2}-1+2 k}$ :

$$
a_{\gamma_{2}-1+2 k}=\frac{(-1)^{k} C_{0}^{k}}{1-C^{\gamma_{2}+2 k}} \frac{\left(\gamma_{2}+2 k\right)\left(\gamma_{2}+k-1\right) !}{\gamma_{2} ! k !\left(\gamma_{2}-1+2 k\right) !}, \quad k=0,1, \ldots .
$$

Подставляя найденные функции $\psi_{1}(t)$ и $\psi_{2}(t)$ в формулу $(36)$, получаем, после вычисления интегралов вида (38), выражение для функции $g_{\left(0, \gamma_{2}\right)}(x, y)$ в виде ряда по функциям Бесселя целого порядка:

$$
\begin{aligned}
g_{\left(0, \gamma_{2}\right)}(x, y)= & \sum_{k=0}^{\infty} \frac{C_{0}^{k}}{1-C^{\gamma_{2}+2 k}} \frac{\left(\gamma_{2}+2 k\right)\left(\gamma_{2}+k-1\right) !}{i^{\gamma_{2}} \gamma_{2} ! k !} \\
& \times\left(\left(\sqrt{\frac{x}{y}}\right)^{\gamma_{2}+2 k}-\left(\frac{C}{C_{0}} \sqrt{\frac{y}{x}}\right)^{\gamma_{2}+2 k}\right) J_{\gamma_{2}+2 k}(2 i \sqrt{x y}) .
\end{aligned}
$$

Заменим функции Бесселя интегралами

$$
J_{n}(2 t)=\frac{1}{2 \pi i} \oint_{0^{+}} e^{t(u-1 / u)} \frac{d u}{u^{n+1}}, \quad n=0, \pm 1, \pm 2, \ldots .
$$

Тогда, после замены переменной $u=\sqrt{C_{0} x / y} z$,

$$
\begin{aligned}
& \left(\sqrt{\frac{x}{y}}\right)^{\gamma_{2}+2 k} J_{\gamma_{2}+2 k}(2 i \sqrt{x y}) \\
& =\frac{1}{\left(\sqrt{C_{0}}\right)^{\gamma_{2}+2 k}} \frac{1}{2 \pi i} \oint_{0^{+}} \exp \left\{i \sqrt{C_{0}}\left(x z-\frac{y}{C_{0} z}\right)\right\} \frac{d z}{z^{\gamma_{2}+2 k+1}} .
\end{aligned}
$$

Полагая $u=C \sqrt{y /\left(C_{0} x\right)} z$, получаем

$$
\begin{aligned}
& \left(\frac{C}{C_{0}} \sqrt{\frac{y}{x}}\right)^{\gamma_{2}+2 k} J_{\gamma_{2}+2 k}(2 i \sqrt{x y}) \\
& =\frac{1}{\left(\sqrt{C_{0}}\right)^{\gamma_{2}+2 k}} \frac{1}{2 \pi i} \oint_{0^{+}} \exp \left\{i \sqrt{C_{0}}\left(\frac{C y z}{C_{0}}-\frac{x}{C z}\right)\right\} \frac{d z}{z^{\gamma_{2}+2 k+1}} .
\end{aligned}
$$

Подставляя последние выражения в (39) и заменяя $x$ и $y$ по формулам (33), приходим к незамкнутому решению задачи (31), (5):

$$
\begin{aligned}
& g_{\left(0, \gamma_{2}\right)}\left(z_{1}, z_{2}\right)=\sum_{k=0}^{\infty} \frac{1}{\left(i \sqrt{C_{0}}\right)^{\gamma_{2}}\left(1-C^{\gamma_{2}+2 k}\right)} \frac{\left(\gamma_{2}+2 k\right)\left(\gamma_{2}+k-1\right) !}{\gamma_{2} ! k !} \\
& \quad \times \frac{1}{2 \pi i} \oint_{0^{+}}\left[\operatorname { e x p } \left\{z_{1} \frac{1-\sqrt{1-4 p_{20} p_{02}}}{2 p_{20}} i \sqrt{C_{0}}\left(z-\frac{1}{C z}\right)\right.\right. \\
& \left.+z_{2} i \sqrt{C_{0}}\left(z-\frac{1}{z}\right)\right\}-\exp \left\{z_{1} \frac{1-\sqrt{1-4 p_{20} p_{02}}}{2 p_{20}} i \sqrt{C_{0}}\left(z-\frac{1}{C z}\right)\right. \\
& \left.\left.+z_{2} i \sqrt{C_{0}}\left(C z-\frac{1}{C z}\right)\right\}\right] \frac{d z}{z^{\gamma_{2}+2 k+1}} .
\end{aligned}
$$


Б). Построение замкнутого решения задачи (31), (5). Для ряда (40) можно обосновать законность перестановки знаков суммирования и интегрирования, суммирования и дифференцирования. Справедливы следующие преобразования, при имеющем место условии $C<1$ и $|z|>1$ $\left(\gamma_{2}=1,2, \ldots\right)$ :

$$
\begin{gathered}
\sum_{k=0}^{\infty} \frac{1}{1-C^{\gamma_{2}+2 k}} \frac{\left(\gamma_{2}+k-1\right) !}{\left(\gamma_{2}-1\right) ! k !} \frac{1}{z^{\gamma_{2}+2 k}}=\sum_{k=0}^{\infty} \frac{\left(\gamma_{2}+k-1\right) !}{\left(\gamma_{2}-1\right) ! k !} \frac{1}{z^{\gamma_{2}+2 k}} \sum_{r=0}^{\infty} C^{r\left(\gamma_{2}+2 k\right)} \\
=\sum_{r=0}^{\infty}\left(\frac{C^{r}}{z}\right)^{\gamma_{2}} \sum_{k=0}^{\infty} \frac{\left(\gamma_{2}+k-1\right) !}{\left(\gamma_{2}-1\right) ! k !}\left(\frac{C^{2 r}}{z^{2}}\right)^{k}=\sum_{r=0}^{\infty}\left(\frac{C^{r}}{z}\right)^{\gamma_{2}}\left(1-\frac{C^{2 r}}{z^{2}}\right)^{-\gamma_{2}} .
\end{gathered}
$$

Тогда выражение $(40)$ для $g_{\left(0, \gamma_{2}\right)}\left(z_{1}, z_{2}\right)$ получает вид:

$$
\begin{aligned}
& g_{\left(0, \gamma_{2}\right)}\left(z_{1}, z_{2}\right)=\frac{1}{2 \pi i} \\
& \times \oint_{\theta^{+}}\left[\exp \left\{z_{1} \frac{1-\sqrt{1-4 p_{20} p_{02}}}{2 p_{20}} i \sqrt{C_{0}}\left(z-\frac{1}{C z}\right)+z_{2} i \sqrt{C_{0}}\left(z-\frac{1}{z}\right)\right\}\right. \\
& \left.-\exp \left\{z_{1} \frac{1-\sqrt{1-4 p_{20} p_{02}}}{2 p_{20}} i \sqrt{C_{0}}\left(z-\frac{1}{C z}\right)+z_{2} i \sqrt{C_{0}}\left(C z-\frac{1}{C z}\right)\right\}\right] \\
& \quad \times \frac{1}{-\gamma_{2}} d\left(\frac{1}{\left(i \sqrt{C_{0}}\right)^{\gamma_{2}}} \sum_{r=0}^{\infty}\left(\frac{C^{r} z^{-1}}{1-C^{2 r} z^{-2}}\right)^{\gamma_{2}}\right),
\end{aligned}
$$

в качестве контура интегрирования $\theta$ берем окружность $|z|=1 / \sqrt{C}$. Во втором слагаемом заменим переменную $z$ на $-1 /(C z)$. Получаем

$$
\begin{aligned}
& g_{\left(0, \gamma_{2}\right)}\left(z_{1}, z_{2}\right)=\frac{1}{2 \pi i} \oint_{\theta^{+}} e^{z_{1} s_{1}(z)+z_{2} s_{2}(z)} \\
& \quad \times \frac{(-1)^{\gamma_{2}}}{-\gamma_{2}} d\left(\left(\frac{i}{\sqrt{C_{0}}}\right)^{\gamma_{2}}\left\{\sum_{r=0}^{\infty}\left(\frac{C^{r} z^{-1}}{1-C^{2 r} z^{-2}}\right)^{\gamma_{2}}+\sum_{r=1}^{\infty}\left(\frac{-C^{r} z}{1-C^{2 r} z^{2}}\right)^{\gamma_{2}}\right\}\right) .
\end{aligned}
$$

Сравнивая последнее выражение и (27), получаем замкнутое представление для $g_{\left(0, \gamma_{2}\right)}\left(z_{1}, z_{2}\right)$ :

$$
g_{\left(0, \gamma_{2}\right)}\left(z_{1}, z_{2}\right)=\frac{1}{2 \pi i} \int_{T} e^{z_{1} s_{1}(z)+z_{2} s_{2}(z)} \frac{(-1)^{\gamma_{2}}}{-\gamma_{2}} d f_{\gamma_{2}}(u) ;
$$

из сравнения параметров находим значения периодов $4 \omega, 2 i \omega^{\prime}$ функции $f_{\gamma_{2}}(u)$ и отрезок интегрирования $T$ - данные в формулировке теоремы.

Некоторые из сделанных выше преобразований нуждаются в детальном обосновании их законности; однако непосредственной подстановкой выражения (41) в уравнение (31) и проверкой условий (5) убеждаемся, 
что (41) является решением задачи (31), (5). Из определения экспоненциальной производящей функции (2) и разложения (8) получаем формулу (29) для вероятностей $q_{\left(0, \gamma_{2}\right)}^{\left(\alpha_{1}, \alpha_{2}\right)}$. Теорема 5 доказана.

3 а м е ч а н и е 2. Сравнивая формулу (29) с формулами для $q_{\left(0, \gamma_{2}\right)}^{\left(\alpha_{1}, \alpha_{2}\right)}$, полученными в теоремах 2-4, можно сделать предположение, что $f_{\gamma_{2}}(u)=\left(f_{1}(u)-c_{1}\right)^{\gamma_{2}}+c_{\gamma_{2}}$, где $c_{1}, c_{2}, \ldots$ - некоторые константы.

3 а м е ч а н и е 3 . Показатель первой экспоненты в решении (40) имеет вид $z_{1} s_{1}(z)+z_{2} s_{2}(z)$, где функции $s_{1}(z)$ и $s_{2}(z)$ определены формулами (30). Рациональные функции $s_{1}(z)$ и $s_{2}(z)$ удовлетворяют характеристическому уравнению (32) - являются униформизацией римановой поверхности (6). Легко проверить, что функции

$$
v_{n}\left(z_{1}, z_{2}\right)=\frac{1}{2 \pi i} \oint_{0^{+}} e^{z_{1} s_{1}(z)+z_{2} s_{2}(z)} \frac{d z}{z^{n+1}}, \quad n=0, \pm 1, \pm 2, \ldots
$$

являются решениями уравнения (31). Ряд (40) записывается после замены переменной во втором слагаемом под знаком интеграла в виде ряда

$$
g_{\left(0, \gamma_{2}\right)}\left(z_{1}, z_{2}\right)=\sum_{n=-\infty}^{\infty} a_{n} v_{n}\left(z_{1}, z_{2}\right)
$$

При $h\left(s_{1}, s_{2}\right)=p_{20} s_{1}^{2}+p_{02} s_{2}^{2}+p_{10} s_{1}+p_{01} s_{2}+p_{00}$ или $h\left(s_{1}, s_{2}\right)=$ $p_{22} s_{1}^{2} s_{2}^{2}+p_{21} s_{1}^{2} s_{2}+p_{12} s_{1} s_{2}^{2}+p_{20} s_{1}^{2}+p_{02} s_{2}^{2}$ также можно получить представление решения соответствуюшего уравнения (4) в незамкнутом виде (42), при простой униформизации двулистной римановой поверхности (6), что определяет вывод методами п. 8 интегрального представления вида (9) для вероятностей $q_{\left(0, \gamma_{2}\right)}^{\left(\alpha_{1}, \alpha_{2}\right)}$ в этих случаях.

3 а м е ч а н и е 4. В рассматриваемом специальном случае ветвящегося процесса частицы типа $T_{2}$ называются финальными [2]. Обозначим $\eta^{\left(\alpha_{1}, \alpha_{2}\right)}$ число финальных частиц, которые останутся после того, как процесс выродился, т.е. не останется частиц типа $T_{1}$. Случайная величина $\eta^{\left(\alpha_{1}, \alpha_{2}\right)}$ имеет распределение $\left\{q_{\left(0, \gamma_{2}\right)}^{\left(\alpha_{1}, \alpha_{2}\right)}, \gamma_{2}=0,1, \ldots\right.$; $\left.\sum_{\gamma_{2}=0}^{\infty} q_{\left(0, \gamma_{2}\right)}^{\left(\alpha_{1}, \alpha_{2}\right)} \leqslant 1\right\}$. Полученные формулы для $q_{\left(0, \gamma_{2}\right)}^{\left(\alpha_{1}, \alpha_{2}\right)}$ определяют возможность вывода представляющих интерес для приложений предельных теорем для числа финальных частиц, при условиях $\alpha_{1} \rightarrow \infty, \alpha_{2} \rightarrow \infty([2$, гл. $5, \S 5],[1],[6])$.

Автор благодарен А. Куликовских за ряд ценных указаний.

\section{СПИСОК ЛИТЕРАТУРЫ}

1. Малыщев В.А. Случайные блуждания. Уравнения Винера-Хопфа в четверти плоскости. Автоморфизмы Галуа. М.: Изд-во Моск. ун-та, 1970, 201 с.

2. Севастьянов Б. А. Ветвящиеся процессы. М.: Наука, 1971, 436 с. 
3. Мальшев В.А. Уравнения Винера-Хопфа и их применение в теории вероятностей. - Итоги науки и техники. Сер. Теория вероятн. Матем. статист. Теоретич. киберн., т. 13. М.: ВИНИТИ, 1975, с. 5-35.

4. Севастьянов Б.А., Калинкин А. В. Ветвящиеся случайные процессы с взаимодействием частищ. - Докл. АН СССР, 1982, т. 264, в. 2, с. 306-308.

5. Громак Ю. И., Мальишев В. А. Вероятность попадания в конечное множество при блуждании в квадранте с поглощением на границе. - Международная конференция по теории вероятностей и математической статистике: Тезисы докладов. Вильнюс, 1973, с. 185-186.

6. Могульский A.A., Рогозин Б. А. Случайные блуждания в положительном квадранте. I: Локальные теоремы; II: Интегральные теоремы; III: Константы в интегральной и локальной теоремах. - Матем. труды, 1999, т. 2, № 2, с. 57-97; 2000 , т. 3, № 1, с. 48-118; 2001, т. 4, № 1, с. 68-93.

7. Феллер В. Введение в теорию вероятностей и ее приложения. Т. 1. М.: Мир, 1984, $528 \mathrm{c}$.

8. Two-sex problem. Encyclopedia of Statistical Sciences. V. 9. New York: Wiley, 1988, p. 373.

9. Сидоров Ю.В., Федорюк М. В., Шабунин М. И. Лекции по теории функций комплексного переменного. М.: Наука, 1989, $480 \mathrm{c}$.

10. Евграфов М. А. Аналитические функции. М.: Наука, 1965, 424 с.

11. Паламодов В. П. Линейные дифференциальные операторы с постоянными коэффициентами. М.: Наука, 1967, 488 с.

12. Уиттекер Э. Т., Ватсон Д. Н. Курс современного анализа. Ч. 2. Трансцендентные функции. М.: Физматгиз, 1963, 516 с.

13. Гурвич A. Теория аналитических и эллиптических функций. Л.: ГТТИ, 1933, $344 \mathrm{c}$.

14. Смирнов М. М. Задачи по уравнениям математической физики. М.: Наука, 1975, $128 \mathrm{c}$.

15. Бичадзе А.В., Калиниченко Д. Ф. Сборник задач по уравнениям математической физики. М.: Наука, 1985, 312 с.

16. Бейтмен $\Gamma$., Эрдейи А. Высшие трансцендентные функции. Функции Бесселя, функции параболического цилиндра, ортогональные многочлены. М.: Наука, $1974,296 \mathrm{c}$.

17. Градштейн И. С., Рыжик И. М. Таблицы интегралов, сумм, рядов и произведений. М.: Наука, 1971, 1108 c.

18. Калинкин $A . B$. Вероятность вырождения ветвящегося процесса с взаимодействием частиц. - Теория вероятн. и ее примен., 1982, т. 27, в. 1, с. 192-197.

19. Калинкин $A . B$. Финальные вероятности для ветвящегося случайного процесса с взаимодействием частиц. - Докл. АН СССР, 1983, т. 269, в. 6, с. 1309-1312.

20. Калинкин $A . B$. Метод экспоненциальной производящей функции для случайных блужданий в четверти плоскости. - Докл. РАН, 2000, т. 275, в. 5, с. 583-587.

Поступила в редакцию

8.IX.2000 